\title{
CONSENSUS BASED ON MULTIPLICATIVE CONSISTENT DOUBLE HIERARCHY LINGUISTIC PREFERENCES: VENTURE CAPITAL IN REAL ESTATE MARKET
}

\author{
Xunjie GOU ${ }^{1,2}$, Huchang LIAO $^{1,2}$, Xinxin WANG ${ }^{1}$, Zeshui XU ${ }^{1, *}$, Francisco HERRERA ${ }^{2,3}$ \\ ${ }^{1}$ Business School, Sichuan University, Chengdu 610064, China \\ 2 Andalusian Research Institute in Data Science and Computational Intelligence (DaSCI), \\ University of Granada, 18071 Granada, Spain \\ ${ }^{3}$ Faculty of Computing and Information Technology, King Abdulaziz University, Jeddah, Saudi Arabia
}

Received 29 March 2019; accepted 28 April 2019

\begin{abstract}
Based on the Computing with Words (CW), double hierarchy hesitant fuzzy linguistic term set (DHHFLTS) can be used to express complex linguistic information accurately with two simple linguistic hierarchies. This paper proposes a group decision making (GDM) model based on multiplicative consistency and consensus with double hierarchy hesitant fuzzy linguistic preference relations (DHHFLPRs). Firstly, a correlation coefficient of DHHFLTSs is defined based on the distance measures of double hierarchy hesitant fuzzy linguistic elements (DHHFLEs). Then, a multiplicative consistency property of DHHFLPRs is investigated, and a consistency checking method and a feedback mechanism-based repairing algorithm are developed to ensure all DHHFLPRs with acceptable multiplicative consistency. Furthermore, a correlation measure for DHHFLPRs based on the correlation coefficient of DHHFLTSs is proposed, and a new consensus reaching method on the basis of the correlation measure is developed, which can be used to fully obtain the consensus degree from both positive and negative angles. Finally, we make some comparative analyses with other existing consistency checking and repairing method as well as the consensus reaching approach to illustrate the effectiveness of the proposed method by a case study concerning the assessment of the venture capital project about real estate market in some cities of China.
\end{abstract}

Keywords: double hierarchy hesitant fuzzy linguistic preference relations, group decision making, multiplicative consistency, consensus, venture capital, real estate market.

\section{Introduction}

Group decision making (GDM), ranking a finite set of alternatives based on the evaluations of a group of decision makers (DMs), has attracted many scholars' attention in recent years. In general, a complete GDM process mainly consists of three aspects: a) evaluation process; b) consensus reaching process; c) alternatives ranking process. In evaluation process, natural languages can reflect $\mathrm{DMs}$ original evaluation information better when they provide preferences. For instance, when evaluating a car, there's a high probability that the first thing we tend to do is to say some words like "the performance is very good" and "the price is cheap" rather than some quantitative expressions. Therefore, fuzzy linguistic approach was proposed by Zadeh (1975) to deal with natural languages and he also gave the concept of Computing with Words $(\mathrm{CW})(\mathrm{Za}-$ deh, 2012) that is a system of computation where the ob- jects of computation are words, phrases and propositions drawn from a natural language. Motivated by $\mathrm{CW}$, and as a cognitive complex linguistic information representation tool, the Double Hierarchy Linguistic Term Set (DHLTS) (Gou, Liao, Xu, \& Herrera, 2017) not only can be used to express cognitive complex linguistic information accurately with two simple linguistic hierarchies, but also can be more clear to express the semantics of original natural languages because all elements in a DHLTS are expressed by linguistic labels without any numerical scales. In addition, to represent some hesitant linguistic information, Gou et al. (2017) extended DHLTS into hesitant fuzzy environment and defined the concept of double hierarchy hesitant fuzzy linguistic term set (DHHFLTS). Because of the flexibility and comprehensiveness of the DHLTS and DHHFLTS, they have attracted more and more attention including decision making methodologies (Gou et al.,

*Corresponding author. E-mail: xuzeshui@263.net 
2017; Montserrat-Adell, Xu, Gou, \& Agell, 2019; Krishankumar, Subrajaa, Ravichandran, Kar, \& Saeid, 2019), preference relations (Gou, Liao, Xu, Min, \& Herrera, 2019; Gou, $\mathrm{Xu}, \&$ Herrera, 2018a) and measure methodologies (Gou, Xu, Liao, \& Herrera, 2018b).

In recent years, the pairwise comparison methods are more accurate than non-pairwise methods (Millet, 1997), and the main advantage of pairwise comparison is that DMs only need to focus exclusively on two alternatives at a time when expressing their preferences (Chiclana, Herrera-Viedma, Alonso, \& Herrera, 2009). Then a large number of preference relations have been developed on the basis of different information representation forms, including fuzzy preference relations (Orlorski, 1978; Chiclana, Herrera-Viedma, Herrera, \& Alonso, 2007), multiplicative preference relations (Saaty, 1980), linguistic preference relations (Herrera, Herrera-Viedma, \& Verdegay, 1996), intuitionistic fuzzy preference relations (Xu, 2007, 2008), probabilistic linguistic preference relations (Zhang, Xu, Wang, \& Liao, 2016; Luo, Zhang, Wang, \& Li, 2019; Zhou \& Xu, 2018), hesitant fuzzy preference relations (Zhu, Z. S. Xu, \& J. P. Xu, 2014), hesitant fuzzy linguistic preference relations ( $\mathrm{Wu} \& \mathrm{Xu}, 2016$; $\mathrm{Zhu} \&$ $\mathrm{Xu}, 2014$ ), and 2-tuple fuzzy linguistic preference relation (X. Y. Zhang, H. Y. Zhang, \& Wang, 2019), among others. Considering that the DHHFLTS can reflect the real thought of DMs more completely and the advantage of the preferences discussed above, Gou et al. (2018a) defined the concept of double hierarchy hesitant fuzzy linguistic preference relation (DHHFLPR) and used it to express the assessments of DMs in large-scale group decision making.

However, this way of providing preferences limits DMs in their global perception of the alternatives, generates lots of unnecessary information, and, as a consequence, the provided preferences could be inconsistent (Chiclana et al., 2009). Therefore, measuring consistency is an important step in decision making with each kind of preference relation to ensure that the preferences of DMs are neither random nor illogical. In most preference relations, the research of consistency is usually associated with the notion of transitivity. Take fuzzy preference relation as an example, it mainly has six transitivity conditions including weak transitivity, max-min transitivity, max-max transitivity, restricted max-min transitivity, restricted max-max transitivity, multiplicative transitivity and additive transitivity (Chiclana et al., 2007; Li, Wang, \& Hu, 2019). In existing research, scholars are more inclined to utilize multiplicative transitivity considering that it is a special case of the cycle transitivity property (Baets, Meyer, Schuymer, \& Jenei, 2006). In addition, amounts of scholars have proved that the multiplicative transitivity is the most appropriate property for modeling cardinal consistency of preference relations because it can avoid some gaps such as the conflict with the given range used for providing the preference values (Chiclana et al., 2009). Therefore, the multiplicative consistency has been studied in different preferences such as the multiplicative consistent intuitionistic preference relation (Xu, Cai, \& Szmidt, 2011), the multiplicative consistent fuzzy preference relations (Xia, $\mathrm{Xu}, \&$ Chen, 2013), the perfect multiplicative consistent interval reciprocal relation (Xia \& Xu, 2011), the multiplicative consistent linguistic preference relation (Xia, Xu, \& Wang, 2014), the multiplicative consistent hesitant fuzzy preference relation (Zhu et al., 2014; Li et al., 2019), and multiplicative consistent probabilistic hesitant fuzzy preference relation (Li \& Wang, 2018).

As the discussion in Gou et al. (2017), the DHLTS and DHHFLTS can be regarded as the extensions of fuzzy set in linguistic environment and the transformations between each other are equivalent (Yu, Xu, \& Wang, 2018). Therefore, the advantages of the multiplicative consistency of fuzzy preference relations even other preference relations also work for DHHFLPRs. Based on this, the first contribution of this paper is to focus on investigating the multiplicative consistency of DHHFLPRs. As we mentioned above, it is unrealistic that the DHHFLPRs provided by DMs are perfectly multiplicative consistent. To solve this defect, this paper develops a concept of acceptable multiplicative consistent DHHFLPR, and proposes a consistency checking method to judge whether a DHHFLPR is of acceptable consistency. Additionally, to fully respect and consider the opinions of DMs, a feedback mechanism-based repairing method is developed to improve the consistency of a DHHFLPR.

After ensuring that all DHHFLPRs are of acceptable consistencies, we cannot overlook another important step: the consensus reaching process, which is an essential process in GDM for enabling sufficient communications among all DMs and obtaining an accepted decision result. Therefore, the second contribution is to manage the consensus reaching process and ensure that all DMs reach consensus. In existing research, Gou et al. (2018a) developed a consensus reaching method for large-scale group decision-making problems with DHHFLPRs based on similarity measures. In most research, the similarity and distance measures between individual evaluations and group opinions have been used widely to measure the consensus degrees in GDM problems (Liao, Xu, Zeng, \& Xu, 2015; Wu \& Xu, 2016). However, these two kinds of measures can only obtain the positive values which is regarded as the positive correlation coefficients. However, one most important defect of them is that they cannot be used to fully reflect the real relationships between individual evaluations and group opinions by considering only the positive correlation coefficients. Therefore, we dedicate to developing a novel correlation measure for DHHFLPRs by considering the positive correlation and negative correlation coefficients simultaneously from the statistical point of view, and then use it to measure the consensus degrees between individuals and collective opinions.

At the theoretical level, we highlight this paper by the following innovative work:

- This paper proposes a multiplicative consistency property of DHHFLPR, and develops a feedback mechanism-based repairing method to improve the consistency of DHHFLPR. 
- This paper develops a novel correlation measure for DHHFLPRs, which contains both positive correlation and negative correlation coefficients simultaneously.

- A new consensus reaching method on the basis of the correlation measure is developed, which makes the consensus reaching process reasonable.

- We make some comparative analyses with other consistency checking and repairing method, and the existing consensus reaching approach.

Additionally, in the opening ceremony of the 2016 World Economic Forum, Premier Li emphasized that it is urgent to make comprehensively deepen reform to promote transformation and upgrading of China's economy. As emphasized by Schumpeter (1934) in innovation theory that innovation is the dynamic of economy, and more importantly, innovation promotes the transformation of economy as well and the venture capital (VC) played an important role in such transformation through innovation (Zhang, Du, \& Tian, 2018). Dutta and Folta (2016) explained that the most important reason why VC is so popular is that projects with VC-backed can get a higher rate of commercialization and are more efficient in innovation than those with non-VC-backed. However, it is clear that the VC firms are facing great risks when they are making huge profits. Therefore, finding a promising project is the first and crucial step for the success of VC. In recent decades, more and more scholars are interested in behavioral decision-making model when dealing with practical VCs. For instance, Gomes and González (2012) and Zhang, Du, and Tian (2018) applied TODIM method into behavioral decision. Zacharakis, Mcmullen, and Shepherd (2007) explained that VCs also plays a primarily role in investment decision. Under uncertain environment, Yazdipour (2011) proved that risk aversion for gains and risk seeking for losses were the appeared risk attitudes of almost all the VCs.

As we mentioned above, as a cognitive complex linguistic information representation tool, DHHFLPR is very useful in GDM under uncertain linguistic environment. Therefore, it can be used as a powerful evaluation carrier to deal with some VC problems in practical. In addition, considering the important degree of both consistency and consensus reaching process, this paper focuses on assess- ing a practical VC project about real estate market in some cities of China with DHHFLPRs by researching the consensus model based on multiplicative consistency.

This paper is organized as follows: Section 1 reviews some basic concepts of DHHFLTS and DHHFLPR. Section 2 develops the multiplicative consistency property of DHHFLPR, and then introduces a feedback mechanismbased consistency repairing method. Section 3 discusses the consensus reaching process based on the correlation measure between DHHFLPRs. Section 4 illustrates a practical GDM problem to show the effectiveness of the proposed method and makes some comparative analyses. Final concluding remarks are provided in last section. A flow chart can be drawn to show the main work of this paper in Figure 1.

\section{Preliminaries}

In this section, we mainly review some existing linguistic models and discuss the concepts of DHHFLTS and DHHFLPR.

\subsection{The concepts of DHLTS and DHHFLTS}

Natural languages are always around our human being and they can be used to reflect the most real thought of people and so they are the essential tools for communications. In the process of GDM, how to represent and deal with natural languages is one of the most important steps. Therefore, Zadeh (2012) introduced a concept of Computing with Words (CW) and explained it with "Computing with words is a system of computation in which the objects of computation are words, phrases and propositions drawn from a natural language. The carriers of information are propositions. It is important to note that Computing with words is the only system of computation which offers a capability to compute with information described in a natural language." Clearly, CW is one of the most effective ways to process natural languages. Motivated by CW, lots of linguistic models have been developed to represent natural languages such as hesitant fuzzy linguistic term set (HFLTS) (Rodríguez, Martínez, \& Herrera, 2012), 2-tuple linguistic model (Herrera \& Martínez, 2000), virtual

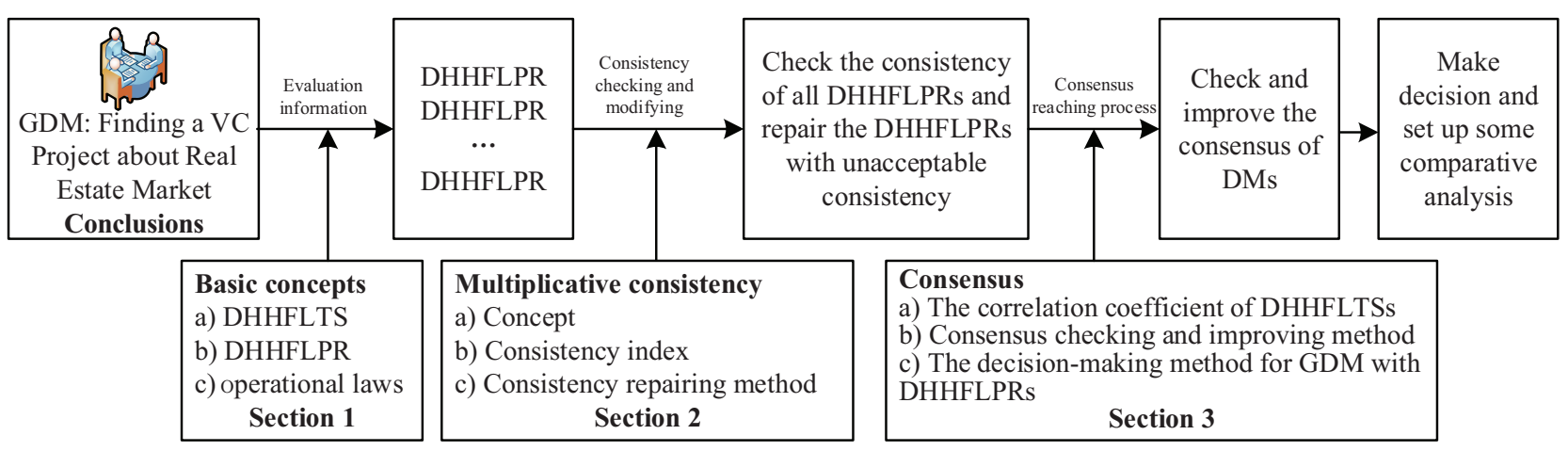

Figure 1. The main work of this paper 
linguistic term model (Xu, 2005), and type-2 fuzzy sets (Mendel, 2007; Qin, Liu, \& Pedrycz, 2017), etc. And most of them are based on symmetrical linguistic term set (LTS) $S=\left\{s_{\alpha} \mid \alpha=-\tau, \ldots,-1,0,1, \ldots, \tau\right\} \quad(\mathrm{Xu}, 2005)$ or asymmetrical LTS $S=\left\{s_{\alpha} \mid \alpha=1,2, \ldots, 2 \tau\right\}$ (Herrera, Herrera-Viedma, \& Verdegay, 1995). For example, HFLTS (Rodríguez et al., 2012) mainly uses several linguistic terms included in a LTS $S$ to express hesitant linguistic information, and 2-tuple linguistic model utilizes both linguistic term and real number to represent linguistic information. However, sometimes the existing linguistic models have many shortcomings. (1) Some linguistic models can be only used to represent one or more basic linguistic terms. For example, let $S=\left\{s_{-2}=\right.$ very low,$s_{-1}=$ low,$s_{0}=$ medium,$s_{1}=$ high, $s_{2}=$ very high $\}$ be a LTS, if we want to express some linguistic information simultaneously as "a little high, just right high, much high", we can only use the basic linguistic term $s_{1}$ to represent it based on $S$ and the modifiers " $a$ little" " just right" and "much" are neglected. Therefore, it is a very important task to enrich each basic linguistic terms of LTS $S$. (2) Some linguistic models have no clear meanings. For example, we cannot explain the real meaning of a virtual linguistic term $s_{1.25}$. Therefore, another important task is to develop method to express this kind of linguistic information clearly. (3) Some linguistic models utilize real numbers to express linguistic information such as 2-tuple linguistic model and type-2 fuzzy set. As we know, quantitative information tends to change the meaning of the original linguistic information.

Apart from above shortcomings, some existing linguistic models provide inspirations and motivations for scholars to create new complex linguistic model. Based on the 2-tuple linguistic structure of 2-tuple linguistic model and type-2 fuzzy set, we can transform all real numbers of 2-tuple linguistic model or type-2 fuzzy sets into linguistic labels and give them real semantics. And then we can obtain a 2-tuple linguistic structure as "adverbs+adjective", as well as utilize the 2-tuple linguistic structure to express linguistic information instead of real numbers. Based on this motivation, Wang, $\mathrm{Xu}$, and Zeng (2018) proposed a concept of linguistic terms with weakened hedges, which regards the "adverbs" as a few weakened hedges expressed by other linguistic labels. However, this linguistic model has two gaps. One is that all weakened hedges are included in a set, which will be inconvenient if different linguistic terms need different sets of weakened hedges. The other one is that a weakened hedge may have different meanings when embellishing different basic linguistic terms but it cannot be expressed clearly. To overcome the gaps of the linguistic terms with weakened hedges and improve 2-tuple linguistic structure, Gou et al. (2017) defined the new concept of DHLTS, which is one of the most typical representatives and it is used to express the 2-tuple linguistic structure based on two hierarchy LTSs, and can be used to express cognitive complex linguistic information accurately with two simple linguistic hierarchies. The first hierarchy LTS is the main linguistic hierarchy and the second hierarchy LTS is the linguistic feature or detailed supplementary of each linguistic term included in the first hierarchy LTS. Let $S=\left\{s_{t} \mid t=-\tau, \ldots,-1,0,1, \ldots, \tau\right\}$ be the first hierarchy LTS and $O^{t}=\left\{o_{k}^{t} \mid k=-\varsigma, \ldots,-1,0,1, \ldots, \zeta\right\}$ be the second hierarchy LTS of $s_{t}$. Then, we call

$S_{O}=\left\{s_{t<o_{k}^{t}>} \mid t=-\tau, \ldots,-1,0,1, \ldots, \tau ; k=-\varsigma, \ldots,-1,0,1, \ldots, \varsigma\right\}$,

the DHLTS, where $s_{t<0_{k}^{t}>}$ is called double hierarchy linguistic term (DHLT). For convenience, we use $O=\left\{o_{k} \mid k=-\varsigma, \ldots,-1,0,1, \ldots, \varsigma\right\}$ and $s_{t<o_{k}>}$ to represent the second hierarchy LTS and DHLT, respectively.

One figure can be drawn to show the DHLTS:

Remark 1. In Figure 2, it is shown that the linguistic term $s_{1}$ has a second hierarchy LTS $O^{1}$. Some advantages of DHLTS can be summarized: 1) All elements in DHLTS are expressed by linguistic labels without any numerical scales, which reflect the semantics of original natural languages to a greater extent; 2) The second hierarchy LTS is necessary when the set of adverbs of a first hierarchy linguistic term is large. Otherwise, we can add them into the first hierarchy LTS directly. 3) Each second hierarchy LTS can be regarded as a set of adverbs and extends the linguistic representations (richer vocabularies). 4) Each linguistic term in the first hierarchy LTS has its own second hierarchy LTS, and all of them are usually different. Especially, considering that these two endpoints $s_{-\tau}$ and

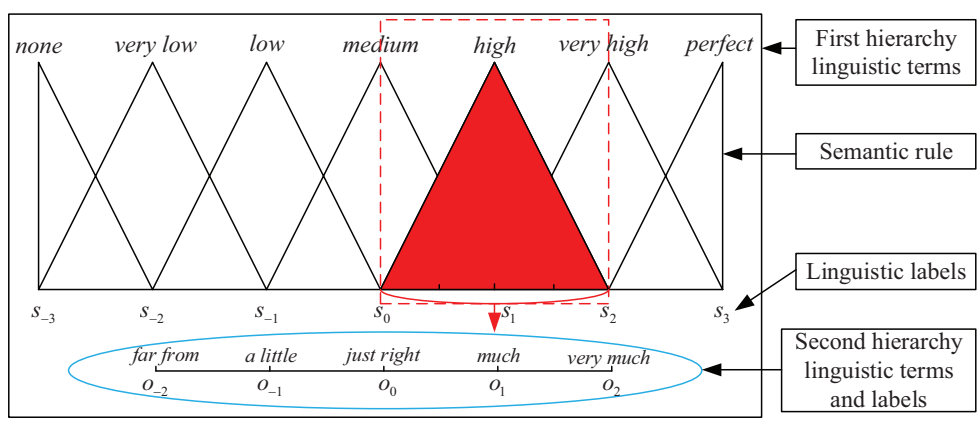

Figure 2. The second hierarchy LTS of a linguistic term in the first hierarchy LTS 
$s_{\tau}$ of the first hierarchy LTS only contain a half of area compared to other linguistic terms, so we can only use $O=\left\{o_{k} \mid k=0,1, \ldots, \varsigma\right\}$ and $O=\left\{o_{k} \mid k=-\varsigma, \ldots,-1,0\right\}$ to be the second hierarchy LTS of them, respectively. More details about DHLTSs can be referred to (Gou et al., 2017).

In addition, to express several complex linguistic terms in a set simultaneously, Gou et al. (2017) extended DHLTS into hesitant fuzzy environment and defined the concept of DHHFLTS, which on $X$, denoted as $H_{S_{O}}$, is in mathematical form of

$$
H_{S_{O}}=\left\{<x_{i}, h_{S_{O}}\left(x_{i}\right)>\mid x_{i} \in X\right\},
$$

where $h_{S_{O}}\left(x_{i}\right)$ is a set of some linguistic terms in $S_{O}$ and can be expressed as

$$
\begin{aligned}
& h_{S_{O}}\left(x_{i}\right)= \\
& \left\{\begin{array}{l}
s_{\phi_{l}<o_{\varphi_{l}}>}\left(x_{i}\right) \mid \begin{array}{l}
s_{\phi_{l}<o_{\varphi_{l}}>} \in S_{O} ; l=1,2, \ldots, L ; \\
\phi_{l} \in\{-\tau, \ldots,-1,0,1, \ldots, \tau\} ; \\
\varphi_{l} \in\{-\varsigma, \ldots,-1,0,1, \ldots, \varsigma\}
\end{array}
\end{array}\right\}
\end{aligned}
$$

with $s_{\phi_{l}<o_{\varphi_{1}}>}$ being the DHLT in $S_{O}$, and $L$ being the number of the DHLTs in $h_{S_{O}}\left(x_{i}\right)$. For convenience, we call $h_{S_{O}}\left(x_{i}\right)$ double hierarchy hesitant fuzzy linguistic element (DHHFLE).

Remark 2. For understanding the DHHFLTS better, its syntax rule was given by establishing a context-free gram$\operatorname{mar} \aleph_{D H H}=\left\{\dot{V}_{N}, \dot{V}_{T}, \dot{I}, \dot{P}\right\}$ as (Gou et al., 2017):

$\dot{V}_{N}=\left\{\begin{array}{l}\langle\text { double hierarchy primary term }\rangle, \\ \langle\text { double hierarchy composite term }\rangle, \\ \langle\text { unary relation }\rangle,\langle\text { binary relation }\rangle,\langle\text { conjunction }\rangle\end{array}\right\}$

$\dot{V}_{T}=\left\{\begin{array}{l}\text { lessthan; morethan; between; and; } \\ s_{-\tau}, s_{1-\tau}, \ldots, s_{0}, \ldots, s_{\tau-1}, s_{\tau} ; o_{-\varsigma}, o_{1-\varsigma}, \ldots, o_{0}, \ldots, o_{\varsigma_{-1}}, o_{\varsigma}\end{array}\right\}$

$\dot{I}=\dot{V}_{N}$.

For the context-free grammar $\aleph_{D H H}$, the production rules $\dot{P}$ can be defined as:

$$
\dot{P}=\left\{\begin{array}{l}
\dot{I}::=\langle\text { double hierarchy primary term }\rangle \\
\mid\langle\text { double hierarchy composite term }\rangle \\
\langle\text { double hierarchy composite term }\rangle::= \\
\langle\text { unary relation }\rangle\langle\text { double hierarchy primary term }\rangle \mid \\
\langle\text { binary relation }\rangle\langle\text { double hierarchy primary term }\rangle \\
\langle\text { conjunction }\rangle\langle\text { double hierarchy primary term }\rangle \\
\langle\text { double hierarchy primary term }\rangle::= \\
s_{-\tau<o_{-\varsigma}}\left|s_{-\tau<o_{-\varsigma+1}}\right| \ldots\left|s_{\tau<o_{\zeta-1}}\right| s_{\left.\tau<o_{\varsigma}\right\rangle} \\
\langle\text { unary relation }\rangle::=\text { less than } \mid \text { more than } \\
\langle\text { binary relation }\rangle::=\text { between } \\
\langle\text { conjunction }\rangle::=\text { and }
\end{array}\right\}
$$

\subsection{The operational laws of DHHFLTSs and the concept of DHHFLPR}

By adding the second hierarchy LTS as the auxiliary linguistic hierarchy, complex linguistic information can be described more clearly. However, some scholars may doubt that whether the calculations among DHHFLTSs is complicated. Here we can explain it and develop some methods to make the calculations simpler.

Motivated by the monotonic function of Dubois (2011) and virtual linguistic terms (Xu, 2005, 2007, 2008), two functions for mutual transformations between DHLT and numerical scale were developed when extending the DHLT to continuous form (Gou et al., 2017). The complexity of the calculation is greatly reduced because of the monotonic function provides convenience for using the mathematical expressions to make the operations among DHLTs. These two functions are shown as follows:

Definition 1 (Gou et al., 2017).

Let $\bar{S}_{O}=\left\{s_{t<o_{k}>} \mid t \in[-\tau, \tau] ; k \in[-\varsigma, \zeta]\right\}$ be a continuous

DHLTS, $h_{S_{O}}=\left\{s_{\phi_{l}<o_{\varphi_{l}}>} \mid s_{\phi_{l}<o_{\varphi_{l}}} \in \bar{S}_{O}\right.$;

$\left.l=1,2, \ldots, L ; \phi_{l} \in[-\tau, \tau] ; \varphi_{l} \in[-\varsigma, \varsigma]\right\}$ be an DHHFLE

with $L$ being the number of DHLTs in $h_{S_{O}}$. Then, the real number $\gamma_{l} \in[0,1]$ and the subscript $\left(\phi_{l}, \varphi_{l}\right)$ of DHLT $s_{\phi_{l}<o_{\varphi_{l}}>}$ can be transformed to each other equivalently by two functions $f$ and $f^{-1}$ :

$$
\begin{aligned}
f: & {[-\tau, \tau] \times[-\varsigma, \varsigma] \rightarrow[0,1], f\left(\phi_{l}, \varphi_{l}\right)=\frac{\varphi_{l}+\left(\tau+\phi_{l}\right) \varsigma}{2 \varsigma \tau}=\gamma_{l} ; } \\
& f^{-1}:[0,1] \rightarrow[-\tau, \tau] \times[-\varsigma, \varsigma], f^{-1}\left(\gamma_{l}\right)= \\
& {\left[2 \tau \gamma_{l}-\tau\right]<o_{\varsigma\left(2 \tau \gamma_{l}-\tau-\left[2 \tau \gamma_{l}-\tau\right]\right)}>=} \\
& {\left[2 \tau \gamma_{l}-\tau\right]+1<o_{\varsigma\left(\left(2 \tau \gamma_{l}-\tau-\left[2 \tau \gamma_{l}-\tau\right]\right)-1\right)}>. }
\end{aligned}
$$

Based on the transformation function $f$, the expected and variance values of an DHHFLE $h_{S_{O}}$ can be defined respectively as:

$$
\begin{aligned}
& E\left(h_{S_{O}}\right)=\frac{1}{\# h_{S_{O}}} \sum_{l=1}^{\# h_{S_{O}}} f\left(s_{\phi_{l}<o_{\varphi_{l}}>}\right) ; \\
& v\left(h_{S_{O}}\right)=\frac{1}{\# h_{S_{O}}} \sum_{l=1}^{\# h_{S_{O}}}\left(f\left(s_{\phi_{l}<o_{\varphi_{l}}}>\right)-E\left(h_{S_{O}}\right)\right)^{2} .
\end{aligned}
$$

For two DHHFLEs $h_{S_{\mathrm{O}_{1}}}$ and $h_{S_{\mathrm{O}_{2}}}$, if $E\left(h_{S_{\mathrm{O}_{1}}}\right)>E\left(h_{S_{\mathrm{O}_{2}}}\right)$, then $h_{S_{O_{1}}} \succ h_{S_{O_{2}}}$. If $E\left(h_{S_{O_{1}}}\right)=E\left(h_{S_{O_{2}}}\right)$, then (1) if $v\left(h_{S_{O_{1}}}\right)>v\left(h_{S_{O_{2}}}\right), h_{S_{O_{2}}} \succ h_{S_{O_{1}}} ;(2)$ if $v\left(h_{S_{O_{1}}}\right)=v\left(h_{S_{O_{2}}}\right)$, then $h_{S_{\mathrm{O}_{1}}} \sim h_{S_{\mathrm{O}_{2}}}$.

Suppose that $h_{S_{O}}=\left\{s_{\phi_{l}<o_{\varphi_{l}}}>\mid s_{\phi_{l}<o_{\varphi_{l}}>} \in S_{O} ; l=1,2, \ldots, \# h_{S_{O}}\right\}$ and 
$h_{S_{O_{i}}}=\left\{s_{\phi_{l}^{i}<o_{\varphi_{l}^{i}}>} \mid s_{\phi_{l}^{i}<o_{\varphi_{l}^{i}}>} \in S_{O} ; l=1,2, \ldots, \# h_{S_{O}}^{i}\right\}$

$\left(i=1,2 ; \# h_{S_{O}}^{1}=\# h_{S_{O}}^{2}\right)$ are three DHHFLEs, and $\lambda(0 \leq \lambda \leq 1)$

is a real number. Some operational laws of DHHFLEs are developed as follows (Gou et al., 2018a):

(1) Addition:

$h_{S_{O_{1}}} \oplus h_{S_{O_{2}}}=\bigcup_{s_{\phi_{l}^{1}<O_{\varphi_{l}>}>} \in h_{S_{O_{1}}}, s_{\phi_{l}^{2}<O_{\varphi_{l}^{2}}>} \in h_{S_{O_{2}}}}\left\{s_{\phi_{l}^{1}+\phi_{l}^{2}<o_{\varphi_{l}^{1}+\varphi_{l}^{2}}>}\right\}$;

if $\phi_{l}^{1}+\phi_{l}^{2} \leq \tau, \varphi_{l}^{1}+\varphi_{l}^{2} \leq \varsigma$;

(2) Multiplication:

$\lambda h_{S_{O}}=\bigcup_{s_{\phi_{l}<o_{\varphi_{l}}}>\in h_{S_{O}}}\left\{s_{\lambda \phi_{l}<o_{\lambda \varphi_{l}}>}\right\} ; \quad 0 \leq \lambda \leq 1 ;$

(3) Complementary operation:

$$
h_{S_{O}}^{c}=\bigcup_{s_{\phi_{l}<o_{\varphi_{l}}}>h_{S_{O}}}\left\{f^{-1}\left(1-f\left(s_{\phi_{l}<o_{\varphi_{l}}}>\right)\right)\right\} \text {. }
$$

In the process of GDM, considering that the pairwise comparison methods are more accurate than non-pairwise methods (Millet, 1997), and the main advantage of pairwise comparison is that of focusing exclusively on two alternatives at a time that facilitates DMs when expressing their preferences (Chiclana et al., 2009). Therefore, under double hierarchy hesitant fuzzy linguistic environment, based on the function $f$ mentioned above, the concept of DHHFLPR is defined as follows:

Definition 2 (Gou et al. 2018a). An DHHFLPR $\tilde{H}_{S_{O}}$ is represented by a matrix $\tilde{H}_{S_{O}}=\left(h_{S_{O_{i j}}}\right)_{m \times m}$, where $h_{S_{O_{i j}}}=\left\{h_{S_{O_{i j}}}^{(l)} \mid l=1,2, \ldots, \# h_{S_{O_{i j}}}\right\} \quad\left(\# h_{S_{O_{i j}}}\right.$ is the number of DHLTs in $h_{S_{O_{i j}}}, h_{S_{O_{i j}}}^{(l)}$ is the $l$-th DHLT in $h_{S_{O_{i j}}}$ ) is an DHHFLE, indicating the hesitant degree of $A_{i}$ over $A_{j}$. For all $i, j=1,2, \ldots, m, h_{S_{O_{i j}}}(i<j)$ satisfies the following conditions:

$$
\begin{aligned}
& f\left(h_{S_{O_{i j}}}^{(l)}\right) \oplus f\left(h_{S_{O_{j i}}}^{(l)}\right)=1, h_{S_{O_{i i}}}=\left\{s_{0<O_{0}>}\right\}, \\
& \# h_{S_{O_{i j}}}=\# h_{S_{O_{j i}}}, h_{S_{O i j}}^{(l)}<h_{S_{O i j}}^{(l+1)} \text { and } h_{S_{O j i}}^{(l)}>h_{S_{O j i}}^{(l+1)} .
\end{aligned}
$$

Additionally, the complement of an DHHFLPR

$$
\tilde{H}_{S_{O}}=\left(h_{S_{O_{i j}}}\right)_{m \times m}=\left(\left\{h_{S_{O_{i j}}}^{(l)} \mid l=1,2, \ldots, \# h_{S_{O_{i j}}}\right\}\right)_{m \times m}
$$

can be denoted as

$$
\tilde{H}_{S_{O}}^{c}=\left(h_{S_{O_{i j}}}^{c}\right)_{m \times m}=\left(\left\{h_{S_{O_{i j}}^{c}}^{c l)} \mid l=1,2, \ldots, \# h_{S_{O_{i j}}}\right\}\right)_{m \times m},
$$

where $h_{S_{O_{i j}}}^{c l)}=f^{-1}\left(1-f\left(h_{S_{O_{i j}}}^{(l)}\right)\right)$.

\section{Multiplicative consistency of DHHFLPR}

This section mainly discusses the multiplicative consistency of DHHFLPR. Firstly, as the basic tool, a normalization method of DHHFLTS is developed, the multiplicative consistency property of DHHFLPRs is defined, and a consistency index of DHHFLPRs is proposed. In addition, a consistency checking method is given to judge whether a DHHFLPR is of acceptable multiplicative consistency. Finally, a feedback mechanism-based method is developed to repair the DHHFLPR with unacceptable multiplicative consistency.

\subsection{The normalization method of DHHFLPR}

To introduce the consistency measure of DHHFLPR, the first step is to normalize DHHFLPR and make sure that all DHHFLEs have the same length. Zhu and Xu (2014) developed two normalization methods, i.e., $\alpha$-normalization and $\beta$-normalization. The former is to remove some elements and the latter is to add some elements. Considering the $\alpha$-normalization may result in more the loss of original information, this paper mainly develops a normalization method for DHHFLPR on the basis of the $\beta$-normalization method.

$$
\text { Let } \tilde{H}_{S_{O}}=\left(h_{S_{O_{i j}}}\right)_{m \times m} \text { be a DHHFLPR, } h_{S_{O_{i j}}^{-}} \text {and } h_{S_{O_{i j}}^{+}}
$$
be the minimum and maximum DHLT obtained by the above comparative method, respectively, $\varepsilon(0 \leq \varepsilon \leq 1)$ be an optimized parameter. Adding $h_{S_{O_{i j}}}^{\prime}=\varepsilon h_{S_{O_{i j}}^{+}}+(1-\varepsilon) h_{S_{O_{i j}}}^{-}$ and $h_{S_{O_{j i}}}^{\prime}=(1-\varepsilon) h_{S_{O_{j i}}^{+}}^{+}+\varepsilon h_{S_{O_{j i}}^{-}}$to $h_{S_{O_{i j}}}$ and $h_{S_{O_{j i}}}$, respectively, then the normalized DHHFLPR can be defined as follows:

\section{Definition 3.}

Let $\tilde{H}_{S_{O}}=\left(h_{S_{O_{i j}}}\right)_{m \times m}=\left(\left\{h_{S_{O_{i j}}}^{(l)} \mid l=1,2, \ldots, \# h_{S_{O_{i j}}}\right\}\right)_{m \times m}$ be an DHHFLPR, $\varepsilon(0 \leq \varepsilon \leq 1)$ be an optimized parameter, and $\widehat{h}_{S_{O_{i j}}}$ be the DHHFLE with the largest number of DHLTs. The normalized DHHFLPR (NDHHFLPR), denoted as $\tilde{H}_{S_{O}}^{N}=\left(h_{S_{O_{i j}}}^{N}\right)_{m \times m}$, can be established by utilizing $\varepsilon$ and $(1-\varepsilon)$ to add the DHLTs to $h_{S_{O_{i j}}}(i<j)$ and $h_{S_{O_{j i}}}(i<j)$, respectively, and we can obtain that the number of DHLTs in all DHHFLEs is equal to $\# \widehat{h}_{S_{O_{i j}}}$, i.e., $\# h_{S_{O_{11}}}^{N}=\# h_{S_{O_{12}}}^{N}$ $=\cdots=\# h_{S_{O_{1 m}}}^{N}=\cdots=\# h_{S_{O_{i j}}}^{N}=\cdots=\# h_{S_{O_{(m-2) m}}^{N}}^{N}=\# h_{S_{O_{(m-1) m}}^{N}}^{N}=\# \widehat{h}_{S_{O_{i j}}}$.

\subsection{Multiplicative consistency of DHHFLPR}

As we mentioned in Section 1, by making pairwise comparison on two alternatives at a time, the opinions of DMs can be represented by the form of preferences. However, this way limits DMs in their global perception of the al- 
ternatives, and the provided preferences may not be rational (Chiclana et al., 2009). In general, the rationality is related to consistency which is associated with the transitivity property. An example can be given to show the transitivity: if alternative $A_{1}$ is preferred to $A_{2}$, and $A_{2}$ is preferred to $A_{3}$, then $A_{1}$ should be preferred to $A_{3}$ through the common sense. Therefore, the lack of consistency in decision making can lead to inconsistent conclusions (Saaty, 1980).

In some research on the consistency, scholars are more inclined to use multiplicative transitivity considering that it is a special case of the cycle transitivity property (Baets et al., 2006). Therefore, for some other preference relations, multiplicative transitivity property is characterized as the most appropriate property to model and measure consistency (Chiclana et al., 2009). In what follows, the multiplicative consistency of DHHFLPRs is defined:

Definition 4. Given an DHHFLPR $\tilde{H}_{S_{O}}=\left(h_{S_{O_{i j}}}\right)_{m \times m}$ and its NDHHFLPR $\tilde{H}_{S_{O}}^{N}=\left(h_{S_{S_{i j}}^{N}}^{N}\right)_{m \times m}$ with the parameter $\varepsilon(0 \leq \varepsilon \leq 1)$. If, for any $i, j, k=1,2, \ldots, m$,

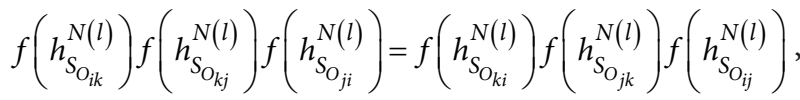

where $h_{S_{O_{i j}}}^{N(l)}$ is the $l$-th DHLT in the DHHFLE $h_{S_{o_{i j}}}^{N}$, then $\tilde{H}_{S_{O}}$ is called a multiplicative consistent DHHFLPR with $\varepsilon(0 \leq \varepsilon \leq 1)$.

Next, two theorems are developed and the proofs of

them are provided in Appendix.
Theorem 1. Given an DHHFLPR $\tilde{H}_{S_{O}}=\left(h_{S_{O_{i j}}}\right)_{m \times m}$ and its NDHHFLPR $\tilde{H}_{S_{O}}^{N}=\left(h_{S_{O_{i j}}}^{N}\right)_{m \times m}$ with the parameter $\varepsilon(0 \leq \varepsilon \leq 1)$, the following statements are equivalent:

I. $\tilde{H}_{S_{O}}$ is multiplicative consistent.

II. $f\left(h_{S_{O_{i j}}^{N(l)}}^{N}\right)=$

$$
\begin{gathered}
\frac{f\left(h_{S_{O_{i k}}}^{N(l)}\right) f\left(h_{S_{O_{k j}}^{N}}^{N(l)}\right)}{f\left(h_{S_{O_{i k}}^{N(l)}}^{N(l)}\right) f\left(h_{S_{O_{k j}}}^{N(l)}\right)+\left(1-f\left(h_{S_{O_{i k}}^{N(l)}}\right)\right)\left(1-f\left(h_{S_{O_{k j}}^{N(l)}}\right)\right)}, \\
i, j, k=1,2, \ldots, m .
\end{gathered}
$$

III. $f\left(h_{S_{O_{i j}}^{N}}^{N(l)}\right)=$

$$
\frac{\sqrt[m]{\prod_{k=1}^{m} f\left(h_{S_{O_{i k}}}^{N(l)}\right) f\left(h_{S_{O_{k j}}}^{N(l)}\right)}}{\sqrt[m]{\prod_{k=1}^{m} f\left(h_{S_{O_{i k}}}^{N(l)}\right) f\left(h_{S_{O_{k j}}}^{N(l)}\right)}+\sqrt[m]{\prod_{k=1}^{m}\left(1-f\left(h_{S_{S_{i k}}}^{N(l)}\right)\right)\left(1-f\left(h_{\left.\left.S_{O_{k j}}^{N(l)}\right)\right)}^{m}\right.\right.}},
$$

Based on Eq. (10), it can be easily proven that

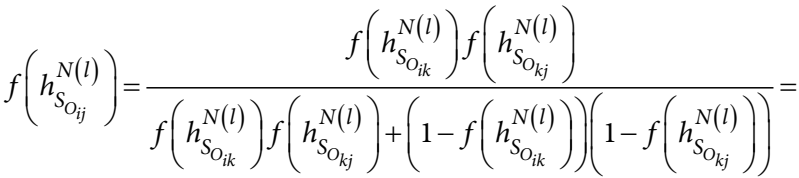

$$
\begin{aligned}
& 1 /\left(1+\left(\frac{1}{f\left(h_{S_{O_{i k}}^{N(l)}}\right)}-1\right)\left(\frac{1}{f\left(h_{S_{O_{k j}}^{N(l)}}^{N(j)}\right.}-1\right)\right) \text {. }
\end{aligned}
$$

Therefore, $f\left(h_{S_{O_{i j}}^{N}}^{N(l)}\right)$ is an increasing function about $f\left(h_{S_{O_{i k}}}^{N(l)}\right)$ and $f\left(h_{S_{O_{k j}}}^{N(l)}\right)$, and we can obtain

$$
0 \leq 1 /\left(1+\left(\frac{1}{f\left(h_{S_{O_{i k}}^{N(l)}}\right)}-1\right)\left(\frac{1}{f\left(h_{S_{O_{k j}}^{N(l)}}\right)}-1\right) \leq 1,\right.
$$

which means that Eq. (10) produces a reasonable result because the result of $f\left(h_{S_{O_{i j}}^{N}}^{N(l)}\right)$ is included in $[0,1]$.

Theorem 2. Given an DHHFLPR $\tilde{H}_{S_{O}}=\left(h_{S_{O_{i j}}}\right)_{m \times m}$ and its NDHHFLPR $\tilde{H}_{S_{O}}^{N}=\left(h_{S_{O_{i j}}}^{N}\right)_{m \times m}$ with the parameter $\varepsilon(0 \leq \varepsilon \leq 1)$, for $i, j, k=1,2, \ldots, m$, let

$\hat{h}_{S_{o_{j}}}^{N(l)}=f^{-1}\left(\frac{\sqrt[m]{\prod_{k=1}^{m} f\left(h_{S_{o_{k}}}^{N(l)}\right) f\left(h_{S_{o_{j}}}^{N(l)}\right)}}{\sqrt[m]{\prod_{k=1}^{m} f\left(h_{S_{O_{k}}}^{N(l)}\right) f\left(h_{S_{o_{j}}}^{N(l)}\right)}+\sqrt[m]{\prod_{k=1}^{m}\left(1-f\left(h_{S_{o_{k}}}^{N(l)}\right)\right)\left(1-f\left(h_{S_{o_{j}}}^{N(l)}\right)\right)}}\right)$.

Then, $\hat{H}_{S_{O}}=\left(\hat{h}_{S_{O_{i j}}^{N}}\right)_{m \times m}$ is a multiplicative consistent DHHFLPR with $\varepsilon$.

Remark 3. The Theorem 1 mainly discusses some properties of the multiplicative consistency of DHHFLPR and the Theorem 2 gives a method to calculate the multiplicative consistent DHHFLPR. Therefore, based on the normalization method and Theorem 2, it is easy to obtain the multiplicative consistent DHHFLPR from an original DHHFLPR. And then we can check and repair the DHHFLPR with unacceptable consistency, which will be discussed in Subsections 2.3 and 2.4.

\subsection{Consistency index for DHHFLPR}

Under most circumstances, the consistency of the DHHFLPR provided by the DM may be unacceptable. Thus, it is important to check and repair the DHHFLPR with unacceptable consistency. Here, we define an acceptably consistent DHHFLPR. To do so, a consistency index for DHHFLPRs is defined. Firstly, the distance between two DHHFLPRs is defined as follows:

Definition 5. Let

$\tilde{H}_{S_{O}}^{k}=\left(h_{S_{O_{i j}}}^{k}\right)_{m \times m}=\left(\left\{h_{S_{O_{i j}}}^{k(l)} \mid l=1,2, \ldots, \# h_{S_{O_{i j}}^{k}}^{k}\right\}\right)_{m \times m}(k=1,2)$ 
be two DHHFLPRs, and $\tilde{H}_{S_{O}}^{k N}=\left(h_{S_{O_{i j}}}^{k N}\right)_{m \times m}=$ $\left(\left\{h_{S_{O_{i j}}}^{k N(l)} \mid l=1,2, \ldots, \# h_{S_{O_{i j}}^{k N}}^{k N}\right\}\right)_{m \times m}(k=1,2)$ be their corresponding NDHHFLPRs with $\varepsilon(0 \leq \varepsilon \leq 1)$, where $\# h_{S_{i j}}^{N}=\# h_{S_{O_{i j}}}^{1 N}=\# h_{S_{O_{i j}}}^{2 N}$. Then the distance between $\tilde{H}_{S_{O}}^{1}$ and $\tilde{H}_{S_{O}}^{2}$ is defined as:

$d\left(\tilde{H}_{S_{O}}^{1}, \tilde{H}_{S_{O}}^{2}\right)=\sqrt{\frac{2}{m(m-1)} \sum_{i<j}^{m}\left(\frac{1}{\# h_{S_{O_{i j}}}^{N}} \sum_{l=1}^{\# h_{S_{i j}}^{N}}\left(f\left(h_{S_{O_{i j}}}^{1 N(l)}\right)-f\left(h_{S_{O_{i j}}}^{2 N(l)}\right)\right)^{2}\right)}$.

Obviously, $d\left(\tilde{H}_{S_{O}}^{1}, \tilde{H}_{S_{O}}^{2}\right)$ satisfies:

(1) $0 \leq d\left(\tilde{H}_{S_{O}}^{1}, \tilde{H}_{S_{O}}^{2}\right) \leq 1$; (2) $d\left(\tilde{H}_{S_{O}}^{1}, \tilde{H}_{S_{O}}^{2}\right)=0$ if and only if $\tilde{H}_{S_{O}}^{1}=\tilde{H}_{S_{O}}^{2}$; (3) $d\left(\tilde{H}_{S_{O}}^{1}, \tilde{H}_{S_{O}}^{2}\right)=d\left(\tilde{H}_{S_{O}}^{2}, \tilde{H}_{S_{O}}^{1}\right)$.

Based on the distance measure of DHHFLPRs, the consistency index of DHHFLPR is defined as follows:

Definition 6. Given a DHHFLPR

$\tilde{H}_{S_{O}}=\left(h_{S_{S_{i j}}}\right)_{m \times m}=\left(\left\{h_{S_{S_{i j}}}^{(l)} \mid l=1,2, \ldots, \# h_{S_{S_{i j}}}\right\}\right)_{m \times m}$, its NDHHFLPR $\tilde{H}_{S_{O}}^{N}=\left(h_{S_{O_{i j}}^{N}}^{N}\right)_{m \times m}=\left(\left\{h_{S_{O_{i j}}^{N(l)}} \mid l=1,2, \ldots, L\right\}\right)_{m \times m}$ with $\varepsilon(0 \leq \varepsilon \leq 1)\left(L=\max \left\{\# h_{S_{O_{i j}}} \mid i, j=1,2, \ldots, m ; i \neq j\right\}\right.$, and its multiplicative consistent DHHFLPR

$\hat{H}_{S_{O}}=\left(\hat{h}_{S_{O_{i j}}^{N}}\right)_{m \times m}=\left(\left\{\hat{h}_{S_{O_{i j}}^{N}}^{N(l)} \mid l=1,2, \ldots, L\right\}\right)_{m \times m}$ with $\varepsilon$.

Then, the consistency index (CI) of $\tilde{H}_{S_{O}}$ is obtained by

$C I\left(\tilde{H}_{S_{O}}\right)=1-d\left(\tilde{H}_{S_{O}}^{N}, \hat{H}_{S_{O}}\right)=$

$1-\left(\frac{2}{m(m-1)} \sum_{i<j}^{m}\left(\frac{1}{L} \sum_{l=1}^{L}\left(f\left(h_{S_{O_{i j}}^{N(l)}}\right)-f\left(\hat{h}_{S_{O_{i j}}^{N}}^{N(l)}\right)\right)^{2}\right)\right)^{1 / 2}$

$C I\left(\tilde{H}_{S_{O}}\right)$ can be taken as a similarity measure between $\tilde{H}_{S_{O}}$ and its multiplicative consistent DHHFLPR $\hat{H}_{S_{O}}$. Therefore, the larger the value of $C I\left(\tilde{H}_{S_{O}}\right)$ is, the more consistent $\tilde{H}_{S_{O}}$ will be. Especially, $C I\left(\tilde{H}_{S_{O}}\right)=1$ if and only if $\tilde{H}_{S_{O}}$ is a complete multiplicative consistent $\mathrm{DH}$ HFLPR.

From Definition 6, it is obvious that the value of $C I\left(\tilde{H}_{S_{O}}\right)$ is decided by $\varepsilon(0 \leq \varepsilon \leq 1)$, which reflects the DM's risk preferences. Considering that the consistent DHHFLPR will be helpful to obtain meaningful results, a nonlinear optimization model is developed to obtain the optimal $C I\left(\tilde{H}_{S_{O}}\right)$ and the corresponding optimal parameter $\varepsilon$ :
Model 1.

$\max C I\left(\tilde{H}_{S_{O}}\right)=1-d\left(\tilde{H}_{S_{O}}^{N}, \hat{H}_{S_{O}}\right)$

$$
\text { s.t. }\left\{\begin{array}{l}
d\left(\tilde{H}_{S_{O}}^{N}, \hat{H}_{S_{O}}\right)= \\
\left(\frac{2}{m(m-1)} \sum_{i<j}^{m}\left(\frac{1}{L} \sum_{l=1}^{L}\left(f\left(h_{S_{O_{i j}}(l)}\right)-f\left(\hat{h}_{S_{O_{i j}}^{N}}^{(l)}\right)\right)^{2}\right)\right)^{1 / 2} . \\
0 \leq \varepsilon \leq 1
\end{array}\right.
$$

Solving Model 1, we can obtain the optimal parameter $\varepsilon$, the unique multiplicative consistent DHHFLPR $\hat{H}_{S_{O}}$ and the unique consistency index $C I\left(\tilde{H}_{S_{O}}\right)$ with the highest consistency level.

Example 1. Let $S_{O}=\left\{s_{t<o_{k}}>t=-4, \ldots, 4 ; k=-4, \ldots, 4\right\}$ be an DHLTS, $\tilde{H}_{S_{O}}=\left(h_{S_{O_{i j}}}\right)_{4 \times 4}$ be an DHHFLPR as:

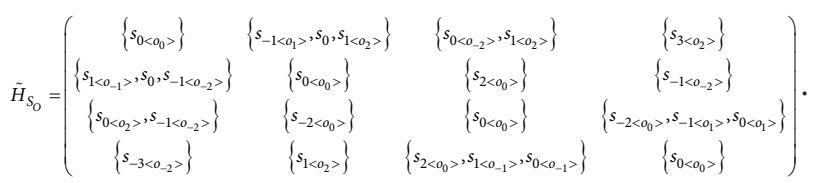

Based on the normalization method with $\varepsilon=1$, we obtain $C I\left(\tilde{H}_{S_{O}}\right)=0.8127$. However, the consistent index of $\tilde{H}_{S_{O}}$ may be different based on different optimized parameter. Therefore, based on Model 1, the variation of the distances between $\tilde{H}_{S_{O}}$ and $\hat{H}_{S_{O}}$ associated to the parameter $\varepsilon$ can be shown in Figure 3. Then we obtain the maximum consistent index of $\tilde{H}_{S_{O}}$ as $C I\left(\tilde{H}_{S_{O}}\right)=0.8127$ with $\varepsilon=1$.

As the calculation results obtained in Example 1, it is common that some DHHFLPRs may be with unacceptable multiplicative consistencies. Then, an acceptably consistent DHHFLPR is developed:

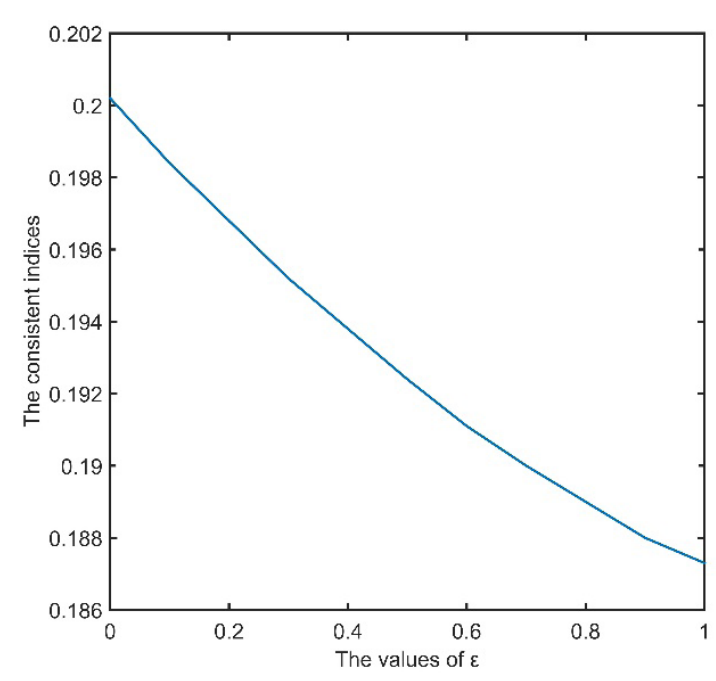

Figure 3. The distances between $\tilde{H}_{S_{O}}$ and $\hat{H}_{S_{O}}$ 
Table 1. The values of consistency thresholds $\overline{C I}\left(\tilde{H}_{S_{O}}\right)$ for different values of $m$ and $T$

\begin{tabular}{|l|l|l|l|l|l|l|}
\hline & $m=3$ & $m=4$ & $m=5$ & $m=6$ & $m=7$ & $m=8$ \\
\hline$T=5$ & 0.8793 & 0.6970 & 0.6512 & 0.6226 & 0.6030 & 0.5888 \\
\hline$T=9$ & 0.8897 & 0.8485 & 0.8256 & 0.8113 & 0.8015 & 0.7944 \\
\hline$T=17$ & 0.9448 & 0.9242 & 0.9128 & 0.9056 & 0.9007 & 0.8972 \\
\hline
\end{tabular}

Definition 7. Let $\tilde{H}_{S_{O}}=\left(h_{S_{O_{i j}}}\right)_{m \times m}$ be an DHHFLPR. Given a threshold value $\overline{C I}$, if

$$
C I\left(\tilde{H}_{S_{O}}\right) \geq \overline{C I}
$$

then we call $\tilde{H}_{S_{O}}$ the DHHFLPR with acceptable multiplicative consistency.

The value of $\overline{C I}$ can be determined based on the DHLTS and the number of the alternatives in GDM. Let $S_{O}=\left\{s_{t<o_{k}>} \mid t=-\tau, \ldots,-1,0,1, \ldots, \tau ; k=-\varsigma, \ldots,-1,0,1, \ldots, \varsigma\right\}$ be an DHLTS, and $T=2 \tau+1$. Gou et al. (2019) proposed the admissible bounds based on the values of $T$ and $m$ for checking the consistency of $\overline{C I}$. However, the threshold values developed by Gou et al. (2019) are based on the distance measure. So, we need to transform them into reasonable values with similarity measure and they are listed in Table 1.

In Example 1, we obtain that $C I\left(\tilde{H}_{S_{O}}\right)=0.8127$. Considering that $T=9$ and $m=4$ we can get $C I\left(\tilde{H}_{S_{O}}\right)=0.8127<\overline{C I}\left(\tilde{H}_{S_{O}}\right)=0.8485$. Therefore, the DHHFLPR discussed in Example 1 is an DHHFLPR with unacceptable multiplicative consistency.

\subsection{The feedback mechanism-based consistency repairing method}

As we mentioned above, it is common that a DHHFLPR $\tilde{H}_{S_{O}}=\left(h_{S_{O_{i j}}}\right)_{m \times m}$ is of unacceptably consistency, i.e., $C I\left(\tilde{H}_{S_{O}}\right)<\overline{C I}\left(\tilde{H}_{S_{O}}\right)$. In this case, we need to repair $\tilde{H}_{S_{O}}$ until it reaches the given threshold. In existing methods, two methods are very popular to improve the consistency of preference relation, i.e., the automatic optimization method (Zhu \& Xu, 2014) and the feedback mechanism (Herrera-Viedma, Alonso, Chiclana, \& Herrera, 2007; Zhu $\& \mathrm{Xu}, 2014)$. The automatic optimization method may lead to unreasonable result because it does not adequately consider the opinions of DMs, and the feedback mechanism can avoid this gap very well. Therefore, this paper develops a feedback mechanism-based consistency repairing method, which can feedback suggestions to DMs and help them to improve their preferences. Before introducing this method, a concept of interval-valued DHHFLTS (IV-DHHFLTS) (Gou et al., 2019) is given:
Definition 8 (Gou et al., 2019). Let $S_{O}$ be a DHLTS. An IV-DHHFLTS on $X, H_{\bar{S}_{\bar{O}}}$, is in mathematical form of

$$
H_{\bar{S}_{\bar{O}}}=\left\{<x_{i}, h_{\bar{S}_{\bar{O}}}\left(x_{i}\right)>\mid x_{i} \in X\right\} \text {, }
$$

where $h_{\bar{S}_{\bar{O}}}=\left\{h_{\bar{S}_{\bar{O}_{i j}}}^{(l)} \mid l=1,2, \ldots, \# h_{\bar{S}_{\bar{O}}}\right\}$. We call $h_{\bar{S}_{\bar{O}}}$ the interval-valued DHHFLE (IV-DHHFLE), and call $h_{\bar{S}_{\bar{O}_{i j}}}^{(l)}$ the interval-valued DHLT (IV-DHLT), where $h_{\bar{S}_{\bar{O}_{i j}}}^{(l)}=\left[\left(h_{\bar{S}_{\bar{O}_{i j}}}^{(l)}\right)^{L},\left(h_{\bar{S}_{\bar{O}_{i j}}}^{(l)}\right)^{U}\right]$ satisfies $\left(h_{\bar{S}_{\bar{O}_{i j}}}^{(l)}\right)^{L} \leq\left(h \bar{S}_{\bar{O}_{i j}}^{(l)}\right)^{U}$.

Based on the IV-DHHFLTS, an IV-DHHFLPR is developed as follows:

Definition 9 (Gou et al., 2019). An IV-DHHFLPR, $\tilde{H}_{\bar{S}_{\bar{O}}}$, is presented by a matrix $\tilde{H}_{\bar{S}_{\bar{O}}}=\left(h_{\bar{S}_{\bar{O}_{i j}}}\right)_{m \times m} \subset A \times A$, where $\quad h_{\bar{S}_{\bar{O}_{i j}}}=\left\{h_{\bar{S}_{\bar{O}_{i j}}}^{(l)} \mid l=1,2, \ldots, \# h_{\bar{S}_{\bar{O}_{i j}}}\right\} \quad$ is an IV-DHHFLE and $h_{\bar{S}_{\bar{O}_{i j}}}^{(i)}$ is the $l$-th IV-DHLT in $h_{\bar{S}_{\bar{O}_{i j}}}$. For all $i, j=1,2, \ldots, m, \quad h_{\bar{S}_{\bar{O}_{i j}}}(i \leq j)$ should satisfy $\left(h_{\bar{S}_{\bar{O}_{i j}}}^{(l)}\right)^{L} \oplus\left(h \bar{S}_{\bar{O}_{j i}}^{(l)}\right)^{U}=\left(h \bar{S}_{\bar{S}_{i j}}^{(l)}\right)^{U} \oplus\left(h_{\bar{S}_{\bar{O}_{j i}}}^{(l)}\right)^{L}=s_{0<o_{0}>}$, $h_{\bar{S}_{\bar{O}_{i i}}}=\left\{s_{0<o_{0}>}\right\}, \quad \# h_{\bar{S}_{\bar{O}_{i j}}}=\# h_{\bar{S}_{\bar{O}_{j i}}}, \quad h_{\bar{S}_{\bar{O}_{i j}}}^{(l)} \leq h_{\bar{S}_{\bar{O}_{i j}}}^{(l+1)}, \quad$ and $h_{\bar{S}_{\bar{O}_{j i}}}^{(l+1)} \leq h_{\bar{S}_{\bar{O}_{j i}}}^{(l)}$.

Then, the feedback mechanism-based consistency repairing method is developed as follows:

Algorithm 1. The feedback mechanism-based consist-

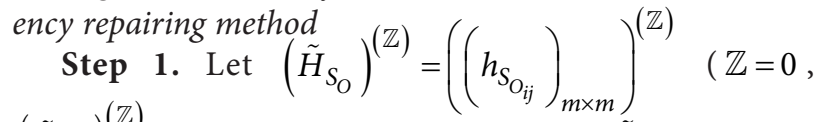
$\left(\tilde{H}_{S_{O}}\right)^{(\mathbb{Z})}$ express the $\mathbb{Z}$-th power of $\tilde{H}_{S_{O}}$, indicating the number of iterations) be a DHHFLPR. Based on Definition 3 and Theorem 2, calculate the NDHHFLPR $\left(\tilde{H}_{S_{O}}^{N}\right)^{(\mathbb{Z})}=\left(\left(h_{S_{O_{i j}}}^{N}\right)_{m \times m}\right)^{(\mathbb{Z})}$ and the multiplicative consistent DHHFLPR $\left(\hat{H}_{S_{O}}\right)^{(\mathbb{Z})}=\left(\left(\hat{h}_{S_{O_{i j}}^{N}}\right)_{m \times m}\right)^{(\mathbb{Z})}$, respec-
tively. 
Step 2. Obtain consistency threshold $\overline{C I}\left(\tilde{H}_{S_{O}}\right)$ based on Table 1.

Step 3. Calculate consistency index $C I\left(\left(\tilde{H}_{S_{O}}\right)^{(\mathbb{Z})}\right)$ based on Eq. (17). If $C I\left(\left(\tilde{H}_{S_{O}}\right)^{(\mathbb{Z})}\right) \geq \overline{C I}\left(\tilde{H}_{S_{O}}\right)$, then go to Step 6; otherwise, go to Step 4.

Step 4. Construct an IV-DHHFLPR $\tilde{H}_{\bar{S}_{\bar{O}}}=\left(\left(h_{\bar{S}_{\bar{O}_{i j}}}\right)_{m \times m}\right)^{(\mathbb{Z})}=\left(\left(\left\{h{\overline{S_{\bar{O}_{i j}}}}(l) \mid l=1,2, \ldots, \# h_{\bar{S}_{\bar{O}_{i j}}}\right\}\right)_{m \times m}\right)^{(\mathbb{Z})}$, where $\left(h_{\bar{S}_{\bar{O}_{i j}}}^{(l)}\right)^{(\mathbb{Z})}=$

$\left[\min \left\{\left(h_{S_{O_{i j}}}^{(l)}\right)^{(\mathbb{Z})}, \hat{h}_{S_{O_{i j}}^{N}}^{N(l)}\right\}, \max \left\{\left(h_{S_{O_{i j}}}^{(l)}\right)^{(\mathbb{Z})}, \hat{h}_{S_{O_{i j}}^{N}}^{N(l)}\right\}\right]$. Take $\tilde{H}_{\bar{S}_{\bar{O}}}$ as a reference and feedback it to the DM, then the $\mathrm{DM}$ provide preference information again, and then go to Step 5.

Step 5. Collect all new preference information and then form the repaired DHHFLPR $\left(\tilde{H}_{S_{O}}\right)^{(\mathbb{Z}+1)}=\left(\left(h_{S_{O_{i j}}}\right)_{m \times m}\right)^{(\mathbb{Z}+1)}$. Let $\mathbb{Z}=\mathbb{Z}+1$. Go back to Step 3.

Step 6. Let $* \tilde{H}_{S_{O}}=\left(\tilde{H}_{S_{O}}\right)^{(\mathbb{Z})}$. Output the repaired normalized DHHFLPR $* \tilde{H}_{S_{O}}$ and end the algorithm.

Considering that the consistency index of the repaired DHHFLPR should be superior to the original DHHFLPR, then an important property about the repaired DHHFLPR can be obtained:

Theorem 3. Given an DHHFLPR $\tilde{H}_{S_{O}}, * \tilde{H}_{S_{O}}$ is the repaired DHHFLPR obtained by Algorithm 1. Then, $C I\left(* \tilde{H}_{S_{O}}\right) \geq C I\left(\tilde{H}_{S_{O}}\right)$.

The proof of Theorem 3 is provided in Appendix. -

\section{A consensus-based decision-making method for GDM with DHHFLPRs}

A general GDM problem mainly consists of a set of alternatives $A=\left\{A_{1}, A_{2}, \ldots, A_{m}\right\}(m \geq 2)$, and a group of DMs $E=\left\{E^{1}, E^{2}, \ldots, E^{Q}\right\}(Q \geq 2)$ with the weight vector $W=\left(w_{1}, w_{2}, \ldots, w_{n}\right)^{T}$. The linguistic preference of DM $E^{q}$ on the degree of the alternative $A_{i}$ being superior to $A_{j}$ is converted to a DHHFLE $h_{S_{O_{i j}}}^{(q)}$. Then all linguistic preferences of $E^{q}$ establish an individual DHHFLPR $\tilde{H}_{S_{O}}^{(q)}=\left(h_{S_{O_{i j}}}^{(q)}\right)_{m \times m}(q=1,2, \ldots, Q)$. When all the individual DHHFLPRs are of multiplicative consistent, the other important step is to make sure that all DMs reach consensus. In this section, firstly the correlation coefficient between any two DHHFLTSs is defined. And then a consensus- based decision-making method is developed with the evaluations expressed by DHHFLPRs. Finally, a decision making method for GDM with DHHFLPRs is established.

\subsection{The correlation coefficient between DHHFLTSs}

Distance measure can reflect the degree of the specific differences between evaluations, and Gou et al. (2018b) introduced some distance measures between DHHFLEs. Suppose that $h_{S_{O_{i}}}=\left\{s_{\phi_{l}^{i}<o_{\varphi_{l}^{i}}>} \mid s_{\phi_{l}^{i}<o_{\varphi_{l}^{i}}>} \in S_{O} ; \quad l=1,2, \ldots, \# h_{S_{O_{i}}}\right\}$ $(i=1,2)$ are two DHHFLEs with $\# h_{S_{O}}=\# h_{S_{\mathrm{O}_{1}}}=\# h_{S_{\mathrm{O}_{2}}}$.

The distance between $h_{\mathrm{S}_{1}}$ and $h_{\mathrm{S}_{2}}$ is defined as:

$d\left(h_{S_{O_{1}}}, h_{S_{O_{2}}}\right)=\sqrt{\frac{1}{\# h_{S_{O}}} \sum_{l=1}^{\# h_{S_{O}}}\left(f\left(s_{\phi_{l}^{1}<o_{\varphi_{l}^{1}}>}\right)-f\left(s_{\phi_{l}^{2}<o_{\varphi_{l}^{2}}>}\right)\right)^{2} .}$

Then the correlation coefficient between two DHHFLTSs $H_{S_{O_{i}}}=\left\{h_{S_{O_{i}}}^{1}, h_{S_{O_{i}}}^{2}, \ldots, h_{S_{O_{i}}}^{m}\right\}(i=1,2)$ is defined as:

$C\left(H_{S_{O_{1}}}, H_{S_{O_{2}}}\right)=$

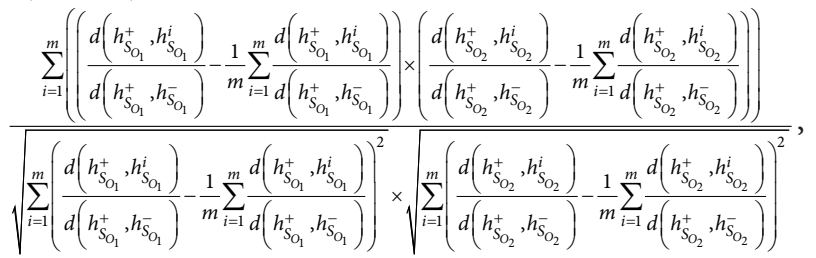

where $m$ is the number of DHHFLEs in each DHHFLTS; $h_{S_{O_{i}}}^{+}$and $h_{S_{O_{i}}}^{-}$are the biggest and smallest DHHFLEs in $H_{S_{O_{i}}}$, respectively, $i=1,2 . d$ is the distance measure by Eq. (18).

Theorem 4. The double hierarchy hesitant fuzzy linguistic correlation coefficient satisfies the property of the Pearson correlation coefficient that $C\left(H_{S_{\mathrm{O}_{1}}}, H_{S_{\mathrm{O}_{2}}}\right) \in[-1,1]$.

The proof of Theorem 4 is provided in Appendix. -

Remark 4. Since $C\left(H_{S_{\mathrm{O}_{1}}}, H_{S_{\mathrm{O}_{2}}}\right) \in[-1,1]$, there exists three situations: (1) If $C\left(H_{S_{\mathrm{O}_{1}}}, H_{S_{\mathrm{O}_{2}}}\right)>0$, then $H_{S_{O_{1}}}$ and $H_{S_{O_{2}}}$ have a positive correlation; (2) If $C\left(H_{S_{\mathrm{O}_{1}}}, H_{S_{\mathrm{O}_{2}}}\right)<0$, then they have a negative correlation; (3) If $C\left(H_{S_{\mathrm{O}_{1}}}, H_{S_{\mathrm{O}_{2}}}\right)=0$, then they have no any correlation. In conclusion, the bigger the absolute value of $\left|C\left(H_{S_{\mathrm{O}_{1}}}, H_{S_{\mathrm{O}_{2}}}\right)\right|$ is, the stronger the correlation between $H_{S_{\mathrm{O}_{1}}}$ and $H_{S_{\mathrm{O}_{2}}}$ should be. Considering that the correlation coefficient reflects the relation of two DHHFLPRs from both positive and negative angles. It can be regarded as a useful tool to represent the consensus degree of DMs in GDM. 


\subsection{Consensus reaching process}

The consensus reaching process mainly consists of two aspects: consensus checking process and consensus improving process.

(a) Consensus checking process

In the GDM process, the consensus measures can be used to depict the closeness degree among the DMs' preferences. The most popular method for measuring the consensus degree is to calculate the deviation degree between each individual preference and aggregated opinion (Dong, $\mathrm{Xu}, \mathrm{Li}$, \& Feng, 2010). Additionally, distance measure is very important to depict the deviations between the DMs' preferences, and it has been utilized to measure the consensus degree in GDM (Gou et al., 2018a). However, the consensus degree measured by distance cannot reflect the relationship between the individual preference and aggregated opinion from both of positive and negative angles, and may lead to some unreasonable consensus results. Motivated by (Wu \& Liao, 2019), and considering that the correlation coefficient of DHHFLTSs discussed in Eq. (19) can overcome this gap. Therefore, this subsection develops a method to obtain the consensus degree of each DM based on the correlation coefficient of DHHFLTSs. Firstly, a double hierarchy hesitant fuzzy linguistic weighted average (DHHFLWA) operator is developed to aggregate all DHHFLPRs into a group DHHFLPR.

Definition 10 (Gou et al., 2018a). Let $h_{S_{O_{i j}}^{p}}^{p}=\left\{h_{S_{O_{i j}}^{p(l)}}^{p} \mid l=1,2, \ldots, \# h_{S_{O_{i j}}^{p}}^{p}\right\}(p=1,2, \ldots, P)$ be a set of

DHHFLEs. We call

$\operatorname{DHHFLWA}\left(h_{S_{S_{i j}}^{1}}^{1}, h_{S_{O_{i j}}}^{2}, \ldots, h_{S_{O_{i j}}^{P}}^{P}\right)=\left\{h_{S_{O_{i j}}}^{G(l)} \mid l=1,2, \ldots, \# h_{S_{O_{i j}}^{G}}\right\}$,

the DHHFLWA operator, where

$h_{S_{O_{i j}}}^{G(l)}=\sum_{p=1}^{P} w_{p} h_{S_{O_{i j}}}^{p(l)}\left(l=1,2, \ldots, \# h_{S_{O_{i j}}^{G}}^{G}\right), w=\left(w_{1}, w_{2}, \ldots, w_{n}\right)^{T}$

is the weight vector of the DHHFLEs and satisfies $0 \leq w_{p} \leq 1$ and $\sum_{p=1}^{P} w_{p}=1$.

Based on Eq. (20), the group DHHFLPR $\tilde{H}_{S_{O}}^{G}=\left(h_{S_{O_{i j}}^{G}}\right)_{m \times m}$ can be established, and we call $h_{S_{O_{i j}}^{G}}$ a group preference element. Then, motivated by Eq. (19), the consensus degree between each DHHFLPR and the group DHHFLPR is developed as follows:

Definition 11. Let $\tilde{H}_{S_{O}}^{(q)}=\left(h_{S_{O_{i j}}}^{(q)}\right)_{m \times m}$ be the individual DHHFLPR of the DM E $E^{q}$, and $\tilde{H}_{S_{O}}^{G}=\left(h_{S_{O_{i j}}^{G}}\right)_{m \times m}$ be the group DHHFLPR. Then the consensus degree of $E^{q}$ is defined as:

$$
\begin{aligned}
& C D\left(\tilde{H}_{S_{O}}^{(q)}\right)=\frac{1}{m} \sum_{j=1}^{m} \Theta_{j}^{(q)} \text {, where } \\
& \Theta_{j}^{(q)}=\frac{\sum_{i=1}^{m}\left(\left(\frac{d_{i j}^{(q)}}{d_{j}^{(q)}}-\frac{1}{m} \sum_{i=1}^{m} \frac{d_{i j}^{(q)}}{d_{j}^{(q)}}\right) \times\left(\frac{d_{i j}^{G}}{d_{j}^{G}}-\frac{1}{m} \sum_{i=1}^{m} \frac{d_{i j}^{G}}{d_{j}^{G}}\right)\right)}{\sqrt{\sum_{i=1}^{m}\left(\frac{d_{i j}^{(q)}}{d_{j}^{(q)}}-\frac{1}{m} \sum_{i=1}^{m} \frac{d_{i j}^{(q)}}{d_{j}^{(q)}}\right)^{2}} \times \sqrt{\sum_{i=1}^{m}\left(\frac{d_{i j}^{G}}{d_{j}^{G}}-\frac{1}{m} \sum_{i=1}^{m} \frac{d_{i j}^{G}}{d_{j}^{G}}\right)^{2}}},
\end{aligned}
$$

where $\quad d_{i j}^{(q)}=d\left(h_{S_{O_{j}}}^{(q)+}, h_{S_{O_{i j}}}^{(q)}\right), \quad d_{j}^{(q)}=d\left(h_{S_{O_{j}}}^{(q)+}, h_{S_{O_{j}}}^{(q)-}\right)$, $d_{i j}^{G}=d\left(h_{S_{O_{j}}}^{G+}, h_{S_{O_{i j}}^{G}}^{G}\right), d_{j}^{G}=d\left(h_{S_{O_{j}}^{G+}}^{G+}, h_{S_{O_{j}}}^{G-}\right), h_{S_{O_{j}}}^{(q)+}=\max _{i} h_{S_{O_{i j}}}^{(q)}$, $h_{S_{O_{j}}}^{(q)-}=\min _{i} h_{S_{O_{i j}}}^{(q)}, h_{S_{O_{j}}^{G+}}^{G+}=\max _{i} h_{S_{O_{i j}}}^{G}$ and $h_{S_{O_{j}}^{G-}}^{G-}=\min _{i} h_{S_{O_{i j}}^{G}}$.

According to Theorem 4, it is obvious that $\Theta_{j}^{(q)} \in[-1,1]$. Then, $C D\left(\tilde{H}_{S_{O}}^{(q)}\right) \in[-1,1]$. If $C D\left(\tilde{H}_{S_{O}}^{(q)}\right)>0$, then, there exists consensus with different strengths. The larger the value of $C D\left(\tilde{H}_{S_{O}}^{(q)}\right)$ is, the stronger the correlation consensus degree of the DM $E^{q}$ to the group will be. However, if $C D\left(\tilde{H}_{S_{O}}^{(q)}\right) \leq 0$, which means negative correlation, so there exists no consensus of $\mathrm{DM} E^{q}$ with respect to the remaining DMs. Based on Karplus and Diederichs, (2012), the strength of consensus degree can be defined in Table 2 .

Table 2. The consensus degree of two DHHFLPRs based on the ranges of correlation strength

\begin{tabular}{|c|c|}
\hline The range of $C D\left(\tilde{H}_{S_{O}}^{(q)}\right)$ & $\begin{array}{c}\text { The correlation strength of two } \\
\text { variables }\end{array}$ \\
\hline$(0.8,1]$ & Extremely strong consensus \\
\hline$(0.6,0.8]$ & Strong consensus \\
\hline$(0.4,0.6]$ & Moderate degree consensus \\
\hline$(0.2,0.4]$ & Weak consensus \\
\hline$(0,0.2]$ & $\begin{array}{c}\text { Extremely weak consensus or no } \\
\text { consensus }\end{array}$ \\
\hline$[-1,0]$ & No consensus \\
\hline
\end{tabular}

(b) Consensus modifying process

After obtaining the consensus degrees of all DMs, there may be some preferences with low consensus degrees. Therefore, it is necessary to improve the group consensus degree by a consensus modifying process before making decision. In this regard, two processes should be handled: one is to detect the individual DHHFLPR that with the lowest consensus degree, and the other is to adjust the corresponding element of it. Considering that the 
automatic modification is a low cost but effective technique (Dong \& Cooper, 2016; Ma, 2016), this subsection develops an automatic modification method to modify the group consensus degree:

Definition 12. Let the minimum consensus degree of the group be $C D^{*}=C D\left(\tilde{H}_{S_{O}}^{(\varphi)}\right)=\min _{q} C D\left(\tilde{H}_{S_{O}}^{(q)}\right)$ $(q=1,2, \ldots, Q), \xi$ be the given consensus threshold, and $\Theta$ be the given correlation threshold. If $C D^{*}<\xi$, then the corresponding individual DHHFLPR $\tilde{H}_{S_{O}}^{(\varphi)}$ with the minimum consensus degree $C D\left(\tilde{H}_{S_{O}}^{(\varphi)}\right)$ should be adjusted as $\tilde{H}_{S_{O}}^{\prime(\varphi)}=\left(h_{S_{O_{i j}}^{\prime}}^{\prime(\varphi)}\right)_{m \times m}$, where

$$
h_{S_{O_{i j}}^{\prime}}^{(\varphi)}=\left\{\begin{array}{ll}
\frac{1}{2}\left(h_{S_{O_{i j}}}^{(\varphi)} \oplus h_{S_{O_{i j}}^{G}}\right), & \text { if } \Theta_{j}^{(\varphi)}<\Theta \\
h_{S_{O_{i j}}}^{(\varphi)}, & \text { if } \Theta_{j}^{(\varphi)} \geq \Theta
\end{array} .\right.
$$

If the consensus degrees of all DMs are larger than or equal to the given consensus threshold $\xi$, then the final group DHHFLPR $\tilde{H}_{S_{O}}^{*}=\left(h_{S_{O_{i j}}}^{*}\right)_{m \times m}$ is obtained by aggregating all the individual DHHFLPRs.

Remark 5. Based on the strength of consensus degree given in Definition 11, the values of the thresholds $\xi$ and $\Theta$ can be determined as $\xi, \Theta \in[0.4,0.8]$. Additionally, according to the practical situation of GDM, the values of the thresholds $\xi$ and $\Theta$ can be adjusted. If the demand for consensus is strict, then the threshold should be given a higher value; Otherwise, a lower threshold value should be provided.

\subsection{The decision-making method for GDM with DHHFLPRs}

In the process of GDM, when all DHHFLPRs are of acceptable consistencies and the consensus degrees of all DHHFLPRs reach the given consensus threshold, the next step is to make a decision.

Firstly, based on the transformation function $f$, we transform all DHHFLEs of the final group DHHFLPR $\tilde{H}_{S_{O}}^{*}=\left(h_{S_{O_{i j}}}^{*}\right)_{m \times m}$ into the real numbers $\gamma_{i j}$ $(i, j=1,2, \ldots, m)$ by the following formula:

$$
\gamma_{i j}=\frac{1}{\# h_{S_{O_{i j}}}^{*}} \sum_{l=1}^{\# h_{S_{i j}}^{*}} f\left(h_{S_{S_{i j}}}^{*(l)}\right) \text {. }
$$

The final group DHHFLPR can be transformed into a complementary preference relation $\Upsilon=\left(\gamma_{i j}\right)_{m \times m}$. Then, sum up all elements in each row and obtain the overall value of each alternative by

$$
\gamma_{i}=\sum_{j=1}^{m} \gamma_{i j}
$$

Finally, the alternatives can be ranked in descending order according to the values of $\gamma_{i}(i=1,2, \ldots, m)$.

To clarify the proposed multiplicative consistency and consensus-based decision-making method, we summarize the procedure as Algorithm 2 and illustrate it by Figure 4 .

Algorithm 2. The multiplicative consistency and consensus-based decision-making method

Step 1. Collect the linguistic preferences of DMs and establish their individual DHHFLPRs $\tilde{H}_{S_{O}}^{(q)}=\left(h_{S_{O_{i j}}}^{(q)}\right)_{m \times m}$
$(q=1,2, \ldots, Q)$. Go to Step 2.

Step 2. Calculate the consistency index of each DHHFLPR CI $\left(\tilde{H}_{S_{O}}^{(q)}\right)$, and determine the threshold $\overline{C I}$ based on Table 1. If $C I\left(\tilde{H}_{S_{O}}^{(q)}\right) \geq \overline{C I}(q=1,2, \ldots, Q)$, then go to Step 4; otherwise, go to Step 3.

Step 3. Find the DHHFLPR $\tilde{H}_{S_{O}}^{(q)}$ with unacceptable consistency. Utilize Algorithm 1 to repair the unacceptably consistent DHHFLPR. Go back to Step 2 .

Step 4. Obtain the group DHHFLPR $\tilde{H}_{S_{O}}^{G}=\left(h_{S_{O_{i j}}^{G}}\right)_{m \times m}$ by Eq. (20). Let $T=1$. Go to Step 5 .

Step 5. Determine the consensus threshold $\xi$ and the correlation threshold $\Theta$, and calculate the consensus degree $C D\left(\tilde{H}_{S_{O}}^{(q)}\right)$ by Eq. (21). If $C D\left(\tilde{H}_{S_{O}}^{(q)}\right) \geq \xi$, then go to Step 7; Otherwise, go to Step 6.

Step 6. Find out the DHHFLPR $C D^{*}$ with $C D\left(\tilde{H}_{S_{O}}^{(\varphi)}\right)=\min _{q} C D\left(\tilde{H}_{S_{O}}^{(q)}\right)$. Derive its adjusted DHHFLPR $\tilde{H}_{S_{O}}^{\prime(\varphi)}=\left(h_{S_{O_{i j}}^{\prime(\varphi)}}^{(\varphi)}\right)_{m \times m}$. Let $T=T+1$, go back to Step 2.

Step 7. Obtain the final group DHHFLPR $\tilde{H}_{S_{O}}^{*}=\left(h_{S_{O_{i j}}}^{*}\right)_{m \times m}$ by Eq. (20). Go to Step 8 .

Step 8. Obtain the ranking of all alternatives based on Eqs (23) and (24) and end the algorithm.

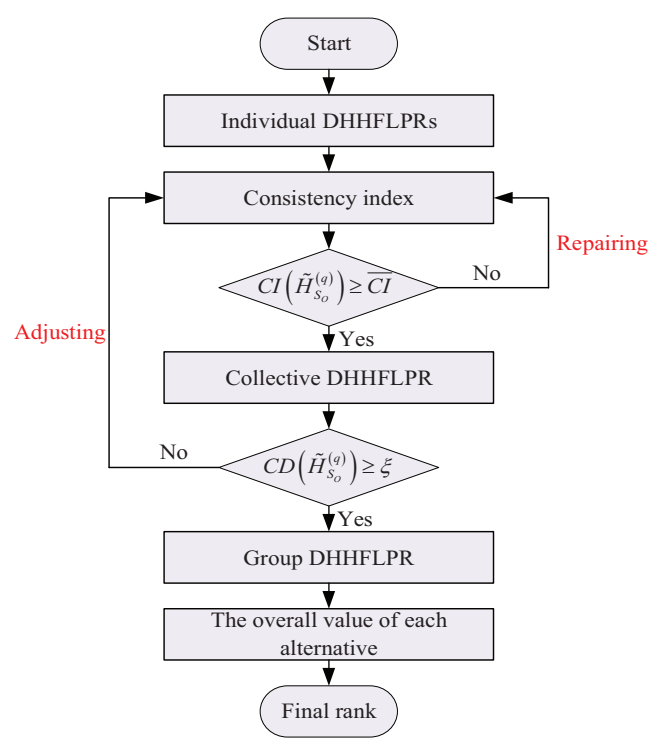

Figure 4. The flowchart of the multiplicative consistency and consensus-based decision-making method 


\section{Case study: finding a VC project about real estate market}

In recent years, especially since 2016 , with the implementation of talent introduction policy and real estate destocking, housing prices have exploded in many Chinese cities such as Nanjing, Chengdu, Xi'an, etc., as well as more and more people believe that property speculation is a huge business opportunity. Meanwhile, lots of real estate developers have sprung up such as Evergrande Real Estate Group, Country Garden Holdings, China Vanke, Greenland, etc. As we know, the real estate market is not only a key part of the Chinese economy but also an integral component of China's financial system. In 2017, housing sales totaled 13.37 trillion RMB, equivalent to $16.4 \%$ of China's GDP (Liu \& Xiong, 2018). However, property speculation has had a huge impact on the real economy. Therefore, holding on to the idea that "house is for living, not for property speculation", each city has given corresponding policies to regulate the housing price. For example, lots of cities' residents cannot buy a house until they meet the requirements of household registration or social security, the central government has adopted steps from raising mortgage rates and down-payments to making buyers pay property tax for the first time. Now, China's economy is also slowing after the government succeeded in reining in credit growth, investment spending and property speculation.

In China, the real estate market can be regarded as a typical VC. In the face of the current real estate market situation, many real estate developers need to consider various factors when making venture investment. For instance, the government's economic regulation and control policy will greatly affect the development direction of the real estate market. In addition, the markets for residential properties and commercial real estate are crucially tied to land sale policies and strategies of local governments (Liu \& Xiong, 2018; Chen, Liu, Xiong, \& Zhou, 2018), so the land prices are also the important factors for the VC in real estate market. Moreover, the households' down payment, the income and price-to-income ratio of mortgage borrowers, and households' housing market expectations. Finally, Firms in China are also heavily exposed to risks of the real estate market. There are at least two key channels that expose firms to the real estate market. One is that the real estate assets are the most widely used collateral for firms to borrow from banks, and the other one is that there is also another speculative channel through which firms actively seek real estate exposure during China's ongoing real estate boom (Liu \& Xiong, 2018; Chen et al., 2018).

Based on these factors, suppose that a firm wants to find a VC project about real estate market in a city of China, and four famous cities are the alternatives as Chengdu $\left(A_{1}\right)$, Nanjing $\left(A_{2}\right)$, Xi'an $\left(A_{3}\right)$ and Zhengzhou $\left(A_{4}\right)$. To do so, four DMs $\left\{E^{1}, E^{2}, E^{3}, E^{4}\right\}$ with the same importance are invited to form a group and to provide their evaluations. Then, the DHHFLPR $\tilde{H}_{S_{O}}^{(q)}$ of each DM can be established based on an DHLTS $S_{O}$, where the first and second LTSs in $S_{O}$ are respectively denoted as:

$S=\left\{s_{-4}=\right.$ extremely bad, $s_{-3}=$ very bad,$s_{-2}=$ bad,$s_{-1}=$ slightly bad, $s_{0}=$ equal, $s_{1}=$ slightly good, $s_{2}=$ good, $s_{3}=$ very good, $s_{4}=$ extremely good $\}$

$O=\left\{o_{-4}=\right.$ far from, $o_{-3}=$ scarcely, $o_{-2}=$ only a littlle, $o_{-1}=$ a little, $o_{0}=$ just right,$o_{1}=$ much,$o_{2}=$ very much, $o_{3}=$ extremely much, $o_{4}=$ entirely $\}$

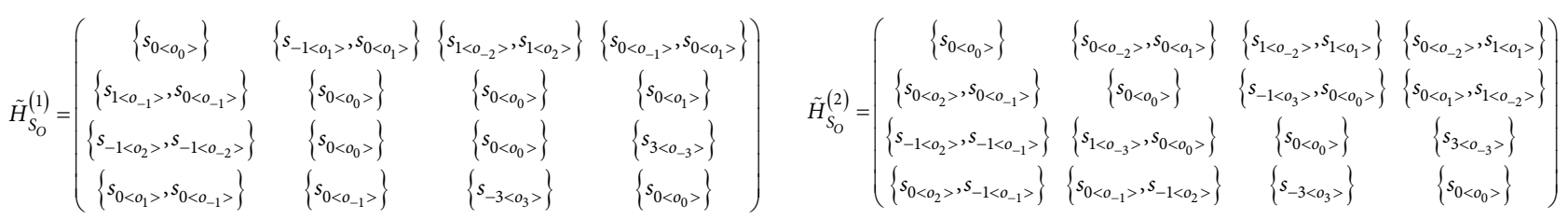

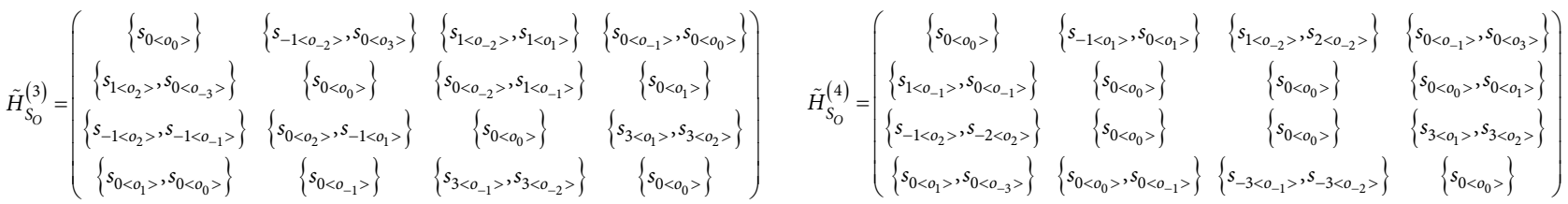

Clearly, this is a GDM problem under double hierarchy hesitant fuzzy linguistic environment. Based on the Algorithm 2, this GDM problem can be solved in Subsection 4.1. 


\subsection{Solving the case by Algorithm 2}

Since Step 1 has been given above, we start to handle this

case from Step 2.
Step 2. Calculate the consistency index $C I\left(\tilde{H}_{S_{O}}^{(q)}\right)$ of each DHHFLPR and show them in Table 3.

Table 3. The consistency index of each DHHFLPR CI $\left(\tilde{H}_{S_{O}}^{(q)}\right)$

\begin{tabular}{|l|l|l|l|l|}
\hline & $\tilde{H}_{S_{O}}^{(1)}$ & $\tilde{H}_{S_{O}}^{(2)}$ & $\tilde{H}_{S_{O}}^{(3)}$ & $\tilde{H}_{S_{O}}^{(4)}$ \\
\hline$C I$ & 0.8967 & 0.9003 & 0.8393 & 0.8502 \\
\hline
\end{tabular}

Based on Table 1, the threshold value is $\overline{C I}=0.8485$. Because $C I\left(\tilde{H}_{S_{O}}^{(3)}\right)<\overline{C I}$, then it is necessary to repair the unacceptably consistent DHHFLPR $\tilde{H}_{S_{O}}^{(3)}$.

Step 3. Utilize Algorithm 1 to repair $\tilde{H}_{S_{O}}^{(3)}$. Firstly, we obtain the IV-DHHFLPR $\tilde{H}_{\overline{S_{\bar{O}}}}^{(3)}$ as:

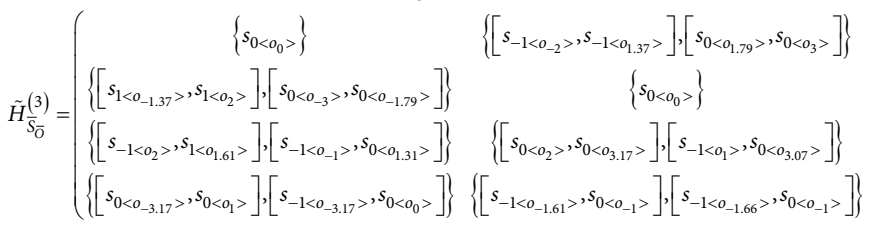

Feed back $\tilde{H}_{\bar{S}_{\bar{O}}}^{(3)}$ to DM $E^{3}$ and ask him to provide preference again based on the suggestions. Then we obtain the new DHHFLPR $\tilde{H}_{S_{O}}^{\prime(3)}$ as:

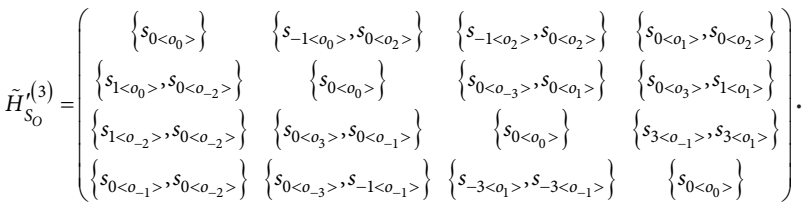

Go back to Step 2, and we obtain $C I\left(\tilde{H}_{S_{O}}^{\prime(3)}\right)=0.9029>\overline{C I}$.

Step 4. Aggregate all DHHFLPRs into a group DHHFLPR $\tilde{H}_{S_{O}}^{G}=\left(h_{S_{O_{i j}}^{G}}^{G}\right)_{m \times m}$ based on Eq. (20):

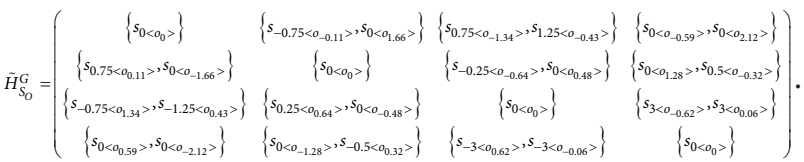

Step 5. Suppose that the consensus threshold $\xi=0.9$ and the correlation threshold $\Theta=0.6$. Calculate the correlation coefficients and consensus degrees of all DMs based on Eq. (21), and the results are listed in Table 4. Clearly, the consensus degree of DM $E^{4}$ is lower than the consensus threshold, and the correlation coefficient $\Theta_{2}$ of $E^{4}$ is lower than the correlation threshold. Therefore, it is necessary to adjust the preference information of $E^{4}$ with respect to the second alternative $A_{2}$.

Table 4. The correlation coefficients and consensus degrees of each DHHFLPR $C I\left(\tilde{H}_{S_{O}}^{(q)}\right)$

\begin{tabular}{|c|c|c|c|c|c|}
\hline \multirow{2}{*}{ DMs } & \multicolumn{4}{|c|}{ Correlation coefficients } & \multirow{2}{*}{$\begin{array}{c}\text { Consensus } \\
\text { degree }\end{array}$} \\
\cline { 2 - 5 } & $\Theta_{1}$ & $\Theta_{2}$ & $\Theta_{3}$ & $\Theta_{4}$ & \\
\hline$E^{1}$ & 0.9700 & 0.8803 & 0.9948 & 0.9970 & 0.9605 \\
\hline$E^{2}$ & 0.9224 & 0.9981 & 0.9969 & 0.9653 & 0.9707 \\
\hline$E^{3}$ & 0.9135 & 0.9917 & 0.9862 & 0.9543 & 0.9614 \\
\hline$E^{4}$ & 0.9998 & -0.5766 & 0.9992 & 0.9863 & 0.6022 \\
\hline
\end{tabular}

$$
\begin{aligned}
& \left\{\left[s_{-1<o_{-1.61}>}>s_{1<o_{-2}>}\right],\left[s_{0<0_{-1.31}>}, s_{1<o_{1}>}\right]\right\} \quad\left\{\left[s_{0<o_{-1}>}>s_{0<0_{3.17}>}\right],\left[s_{0<o_{0}>}, s_{1<o_{3.17}>}\right]\right\} \\
& \left\{\left[s_{0<0_{-3.17}>}, s_{0<0_{-2}>}\right],\left[s_{0<0_{-3.07}>}, s_{1<o_{-1}>}\right]\right\} \quad\left\{\left[s_{0<0_{1}>}>s_{1<0_{1.61}>}>\right],\left[s_{0<0_{1}>}, s_{1<0_{1.66}>}>\right\}\right. \\
& \left\{s_{0<0_{0}>}\right\} \quad\left\{\left[s_{2<0_{0.21}>}, s_{3<0_{1}>}>\right],\left[s_{2<0_{0.18}>}>s_{3<0_{2}>}\right]\right\} \\
& \left\{\left[s_{-3<0_{-1}>}, s_{-2<0_{-0.21}>}\right],\left[s_{-3<0_{-2}>}>s_{-2<0_{-0.18}}>\right]\right\} \quad\left\{s_{0<0_{0}>}\right\}
\end{aligned}
$$

Step 6. Based on Eq. (22), the adjusted DHHFLPR $\tilde{H}_{S_{O}}^{\prime(4)}$ of $E^{4}$ is obtained as follows:

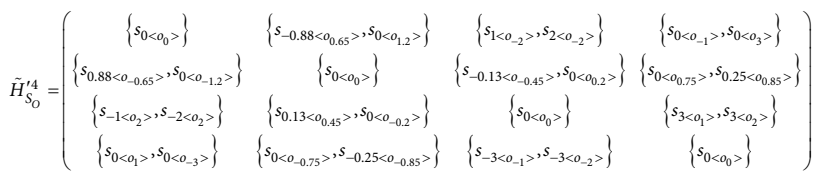

Go back to Step 2. $\tilde{H}_{S_{O}}^{\prime(4)}$ is an DHHFLPR with the acceptable consistency. Additionally, the consensus degree of $\tilde{H}_{S_{O}}^{\prime(4)}$ is $C D\left(\tilde{H}_{S_{O}}^{\prime(4)}\right)=0.9570>0.9$. Therefore, the group preference information reaches the consensus. Therefore, $\tilde{H}_{S_{O}}^{\prime G}$ is the final group DHHFLPR denoted as $\tilde{H}_{S_{O}}^{*}$.

Step 7. Based on Eqs (23) and (24), the ranking of all alternatives is obtained and shown in Table 5. Therefore, the optimal alternative is to invest a VC project about real estate market in Xi'an.

In this subsection, we have checked the consistency of all DHHFLPRs and repaired the DHHFLPR $\tilde{H}_{S_{0}}^{(3)}$. Then, we have also obtained that the consensus degree of DM

\begin{tabular}{|c|c|c|c|c|c|}
\hline \multicolumn{4}{|c|}{ The complementary preference relation } & Overall values & Rank \\
\hline 0.5 & 0.4809 & 0.5984 & 0.5266 & 2.1059 & \multirow{4}{*}{$A_{3} \succ A_{1} \succ A_{2} \succ A_{4}$} \\
\hline 0.5191 & 0.5 & 0.4734 & 0.5723 & 2.0648 & \\
\hline 0.4016 & 0.5266 & 0.5 & 0.8609 & 2.2891 & \\
\hline 0.4734 & 0.4277 & 0.1391 & 0.5 & 1.5402 & \\
\hline
\end{tabular}
$E^{4}$ is lower than the consensus threshold, and have improved it. Especially, the multiplicative consistency and

Table 5. The complementary preference relation and the rank of alternatives 
consensus-based decision-making method proposed in this paper can deal with this case successfully and effectively.

\subsection{Solving the case by additive consistency-based method}

Steps 1-2. Based on the method of Gou et al. (2019), the consistency index of each DM by the additive consistencybased method can be obtained and shown in Table 6 .

$\left.\begin{array}{c}\text { Table 6. The consistency index of each DHHFLPR } C I\left(\tilde{H}_{S_{O}}^{(q)}\right. \\ \text { based on the additive consistent }\end{array}\right)$

\begin{tabular}{|c|c|c|c|c|}
\hline & $\tilde{H}_{S_{O}}^{(1)}$ & $\tilde{H}_{S_{O}}^{(2)}$ & $\tilde{H}_{S_{O}}^{(3)}$ & $\tilde{H}_{S_{O}}^{(4)}$ \\
\hline$C I$ & 0.8975 & 0.9011 & 0.8618 & 0.8619 \\
\hline
\end{tabular}

In Table 6, the preference of each DM is the DHHFLPR with acceptable consistency. Therefore, it is not necessary to make any adjustment.

Step 3. Calculate the group DHHFLPR and the synthetical value of each alternative is obtained as $\{2.0860,2.0469,2.25,1.6172\}$. Then, the ranking of all alternatives is $A_{3} \succ A_{1} \succ A_{2} \succ A_{4}$. Similarly, Xian is also the optimal alternative to invest a VC project about real estate market.

\subsection{Solving the case by the existing consensus reaching method}

Gou et al. (2018a) proposed a consensus reaching method for large-scale GDM with DHHFLPRs. Considering that this method mainly classifies all DMs into several groups and then makes decisions, so each DM discussed in this case can be regarded as a group in (Gou et al., 2018a). Thus, we can use the consensus reaching method proposed in (Gou et al., 2018a) to solve the case.

Steps 1-4. Similar as the Steps1-4 in Section 4.1.

Step 5. Obtain the consensus degrees of the pair of alternatives, the consensus degrees of alternatives and the overall consensus degree of preference relations, and show them in Table 7.

Step 6. Since all DMs reach group consensus, then the synthetical value of each alternative is obtained as $\{2.0860,2.0630,2.2507,1.6004\}$. We can obtain the ranking of all alternatives as $A_{3} \succ A_{1} \succ A_{2} \succ A_{4}$. Xi'an is still the optimal alternative to invest a $\mathrm{VC}$ project about real estate market.

\subsection{Comparative analysis}

All the above three methods show that the ranking of all alternatives are $A_{3} \succ A_{1} \succ A_{2} \succ A_{4}$, which verifies the effectiveness of the proposed method. However, the synthetical value of each alternative, the process of consistency checking and repairing, and the consensus reaching process are different.

(1) The comparative analyses of different consistency properties

In Sections 4.1 and 4.2, we check and repair the consistencies of all DHHFLPRs on the basis of multiplicative consistency and additive consistency, respectively. However, the consistency index of each DHHFLPR obtained by different consistency checking method is different. Especially, by the multiplicative consistency, we can find that $\tilde{H}_{S_{O}}^{(3)}$ is an DHHFLPR with unacceptable multiplicative consistency, but all the DHHFLPRs are with acceptable additive consistency. Therefore, the multiplicative consistency property is much more precise to measure the consistency of DHHFLPR in some degree.

Additionally, we can further use the MATLAB Drawing toolbar to produce the "Figure of area" to give a visible description of the unacceptable consistent DHHFLPRs, the acceptably consistent DHHFLPRs, the multiplicative consistent DHHFLPRs, and the additive consistent DHHFLPRs, which are shown in Figures 5-8. Clearly, both the multiplicative consistent and additive consistent DHHFLPRs are more regular than the original DHHFLPRs. We can also find that the every pair acceptably multiplicative consistent DHHFLPR and the acceptably additive consistent DHHFLPR are very similar, and the multiplicative consistent DHHFLPR has a larger variation range than the additive consistent DHHFLPR in Figures (7-8). Furthermore, the repaired DHHFLPR $\tilde{H}_{S_{O}}^{(3)}$ with acceptable consistency is also more regular than the original DHHFLPR $\tilde{H}_{S_{O}}^{(3)}$ with unacceptable consistency.

(2) Sections 4.1 and 4.3 utilize different consensus reaching methods to deal with the GDM problem. All DMs reach consensus based on Gou's et al. method (Gou et al., 2018a). However, Gou's et al. method only considers the positive consensus degree and ignores the negative consensus degree. Therefore, it cannot reflect the correct result in some degree. In addition, using the proposed method in this paper, we can find that the consensus degree of $E^{4}$ is lower than the consensus threshold, and the correlation coefficient $\Theta_{2}$ of $E^{4}$ is lower than the correlation threshold. Considering the correlation coefficient $\Theta_{2}$

Table 7. Different kinds of consensus degrees

\begin{tabular}{|c|c|c|c|c|c|}
\hline \multicolumn{2}{|c|}{ The consensus degrees of the pair of alternatives } & $\begin{array}{c}\text { the consensus } \\
\text { degrees of } \\
\text { alternatives }\end{array}$ & Overall consensus degree \\
\hline 1 & 0.9607 & 0.9547 & 0.9591 & 0.9582 & \\
\cline { 1 - 5 } 0 & 0.9666 & 0.9639 & 0.9637 & \multirow{2}{*}{0.9442} \\
\hline 0.9607 & 1 & 1 & 0.9112 & 0.9442 & \\
\hline 0.9591 & 0.9666 & 0.9112 & 1 & 0.9447 & \\
\hline
\end{tabular}



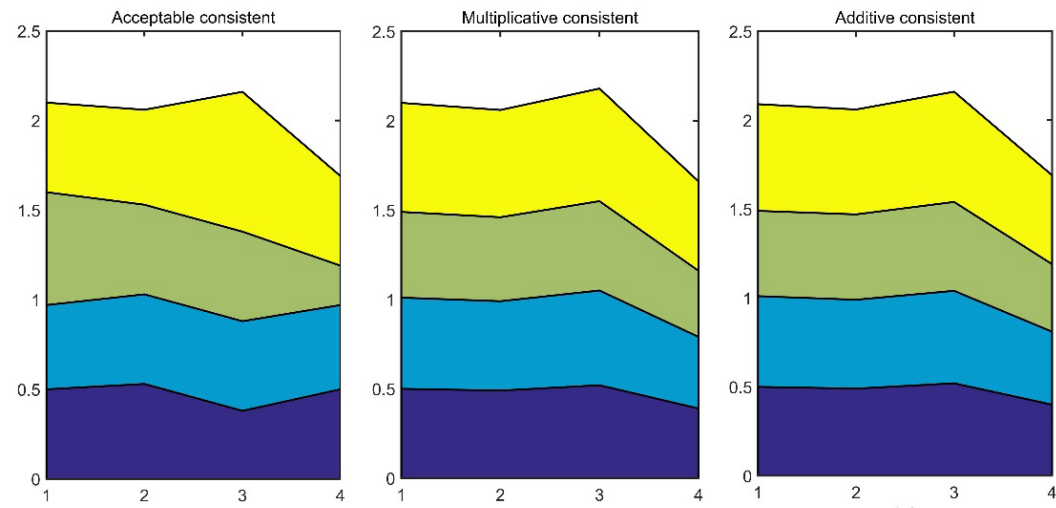

Figure 5. Areas of acceptable, multiplicative and additive consistent DHHFLPR of $\tilde{H}_{S_{O}}^{(1)}$
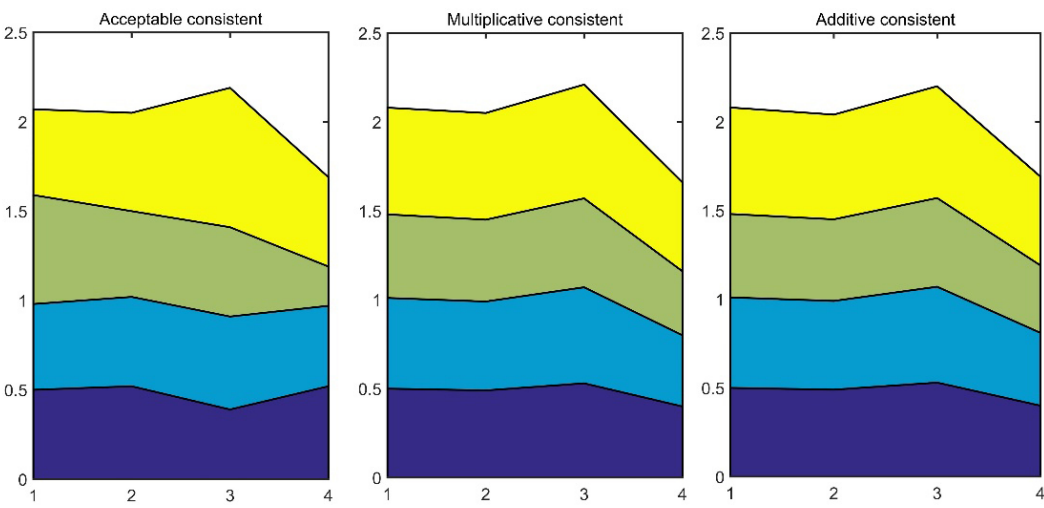

Figure 6. Areas of acceptable, multiplicative and additive consistent DHHFLPR of $\tilde{H}_{S_{O}}^{(2)}$
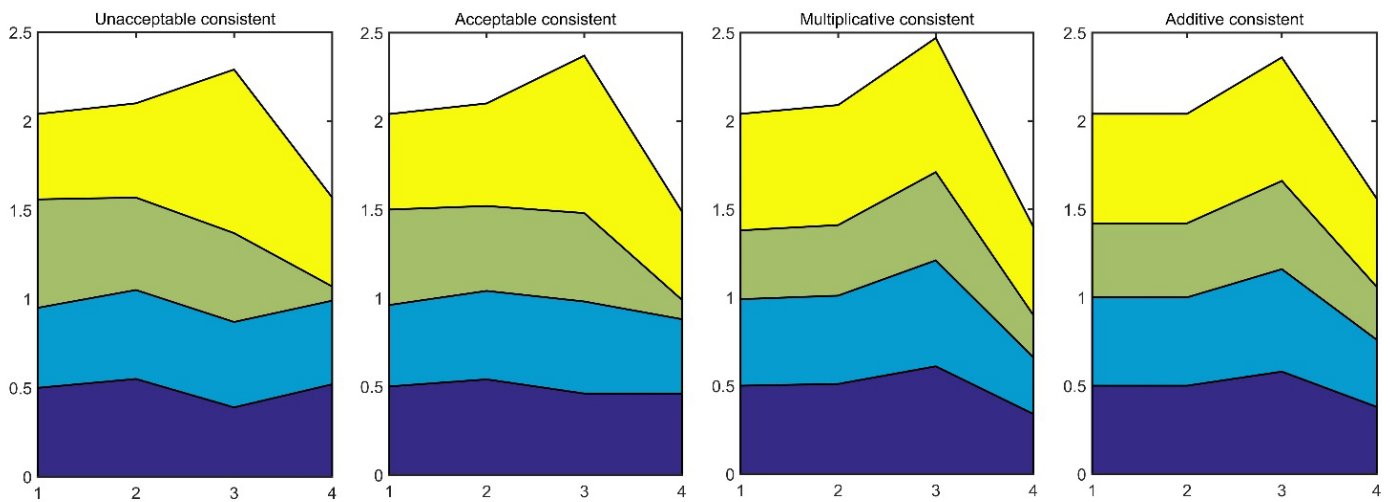

Figure 7. Areas of unacceptable, acceptable, multiplicative and additive consistent DHHFLPR of $\tilde{H}_{S_{O}}^{(3)}$
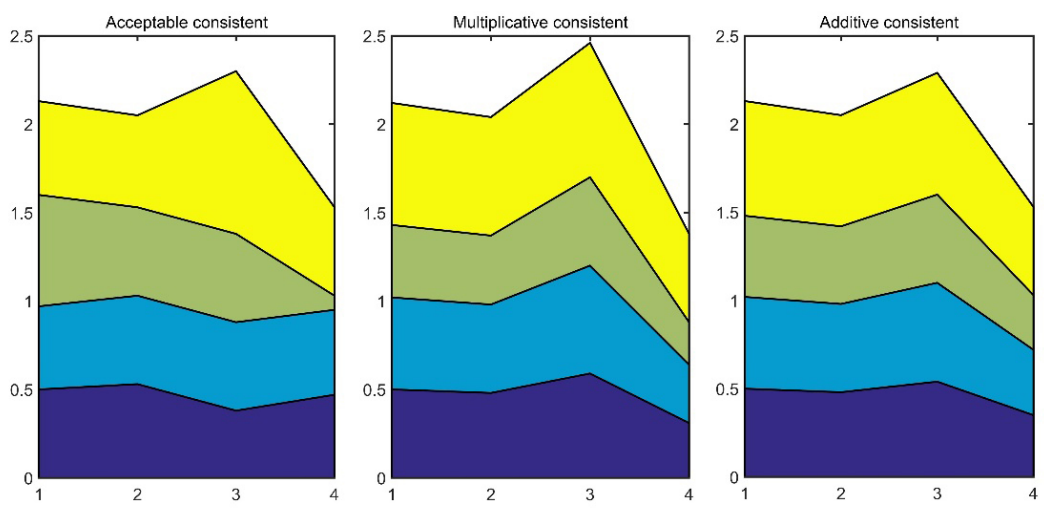

Figure 8. Areas of acceptable, multiplicative and additive consistent DHHFLPR of $\tilde{H}_{S_{O}}^{(4)}$ 
of $E^{4}$ is smaller than 0 , so there exists no consensus by Table 2 and $\mathrm{DM} E^{4}$ needs to repair his preference again. To sum up the above discussion, the correlation coefficient proposed in this paper is within the interval $[-1,1]$, which can be used to depict the strength of consensus degree from the angles of both positive and negative consensus degrees. Therefore, the consensus degree of DHHFLPR based on correlation coefficient is more reasonable than the consensus degree of DHHFLPR based on similarity measure (Gou et al., 2018a).

\section{Conclusions}

This paper has proposed a multiplicative consistency and consensus-based method to solve the GDM problems with DHHFLPRs. We have introduced a multiplicative consistency property of DHHFLPRs, and developed a consistency checking and repairing algorithm to ensure that each DHHFLPR is of acceptable consistency. In addition, a correlation coefficient between DHHFLTSs has been defined based on the distance measure of DHHFLEs, and a new consensus reaching method has also been developed on the basis of the correlation measure for DHHFLPRs. Finally, we have made some comparative analyses with other consistency checking and repairing method, and the existing consensus reaching approach to illustrate the effectiveness of the proposed method by a case study concerning the assessment of the venture capital (VC) projects about real estate market in some cities of China.

In general, the multiplicative consistency and consensus-based model proposed in this paper has the following advantages over the existing methods:

- The correlation coefficient of DHHFLTSs can reflect the positive correlation and negative correlation simultaneously. It is more correct to measure the correlation between two DHHFLTSs than distance and similarity measures.

- The consensus reaching method based on correlation coefficient between DHHFLPRs is more reasonable than some existing information entropy-based measures.

- The multiplicative consistency property is much more precise to measure the consistency of $\mathrm{DHH}$ FLPR in some degree.

As future study, based on the multiplicative consistency of DHHFLPR, some other multiplicative consistency repairing methods can be developed to improve the existing method. Moreover, utilizing the proposed correlation coefficient-based consensus reaching method to solve the problems with other types of preference information would be worthy of study. Additionally, it would be also interesting to implement the proposed multiplicative consistency and consensus-based model to solve other practical GDM problems.

\section{Acknowledgements}

This study was funded by the National Natural Science Foundation of China (Nos. 71571123, 71771155, 71771156 and 71801174), the Major Program of the National Social Science Fund of China (No. 17ZDA092), and the Scholarship from China Scholarship Council (No. 201706240012).

\section{Author contributions}

Xunjie Gou, Huchang Liao and Francisco Herrera conceived the study. Xunjie Gou wrote the first draft of the article. Xinxin Wang were responsible for data collection and analysis. Zeshui Xu were responsible for supervision and funding acquisition.

\section{Disclosure statement}

The authors have no any competing financial, professional, or personal interests from other parties.

\section{References}

Baets, B. D., Meyer, H. D., Schuymer, B. D., \& Jenei, S. (2006). Cyclic evaluation of transitivity of reciprocal relations. Social Choice and Welfare, 26, 217-238.

https://doi.org/10.1007/s00355-006-0093-3

Chen, T., Liu, L., Xiong, W., \& Zhou, L. A. (2017). Real estate boom and misallocation of capital in China (Working paper). Princeton University.

Chiclana, F., Herrera-Viedma, E., Alonso, S., \& Herrera F. (2009). Cardinal consistency of reciprocal preference relations: a characterization of multiplicative transitivity. IEEE Transactions on Fuzzy Systems, 17(1), 14-23.

https://doi.org/10.1109/TFUZZ. 2008.2008028

Chiclana, F., Herrera-Viedma, E., Herrera, F., \& Alonso, S. (2007). Some induced ordered weighted averaging operators and their use for solving group decision-making problems based on fuzzy preference relations. European Journal of Operational Research, 182, 383-399.

https://doi.org/10.1016/j.ejor.2006.08.032

Dong, Q. X., \& Cooper, O. (2016). A peer-to-peer dynamic adaptive consensus reaching model for the group AHP decision making. European Journal of Operational Research, 250(2), 521-530. https://doi.org/10.1016/j.ejor.2015.09.016

Dong, Y. C., Xu, Y. F., Li, H. Y., \& Feng, B. (2010). The OWAbased consensus operator under linguistic representation models using position indexes. European Journal of Operational Research, 203(2), 455-463. https://doi.org/10.1016/j.ejor.2009.08.013

Dubois, D. (2011). The role of fuzzy sets indecision sciences: old techniques and new directions. Fuzzy Sets and Systems, 184, 3-28. https://doi.org/10.1016/j.fss.2011.06.003

Dutta, S., \& Folta, T. B. (2016). A comparison of the effect of angels and venture capitalists on innovation and value creation. Journal of Business Venturing, 31(1), 39-54. https://doi.org/10.1016/j.jbusvent.2015.08.003

Gomes, L. F. A. M., \& González, X. I. (2012). Behavioral multicriteria decision analysis: further elaborations on the TODIM method. Foundations of Computing and Decision Sciences, 37(1), 3-8. https://doi.org/10.2478/v10209-011-0001-1 
Gou, X. J., Liao, H. C., Xu, Z. S., \& Herrera, F. (2017). Double hierarchy hesitant fuzzy linguistic term set and MULTIMOORA method: a case of study to evaluate the implementation status of haze controlling measures. Information Fusion, 38, 22-34. https://doi.org/10.1016/j.inffus.2017.02.008

Gou, X. J., Liao, H. C., Xu, Z. S., Min. R, \& Herrera, F. (2019). Group decision making with double hierarchy hesitant fuzzy linguistic preference relations: consistency based measures, index and repairing algorithms and decision model. Information Sciences, 489, 93-112.

https://doi.org/10.1016/j.ins.2019.03.037

Gou, X. J., Xu, Z. S., \& Herrera, F. (2018a). Consensus reaching process for large-scale group decision making with double hierarchy hesitant fuzzy linguistic preference relations. Knowledge-Based Systems, 157, 20-33.

https://doi.org/10.1016/j.knosys.2018.05.008

Gou, X. J., Xu, Z. S., Liao, H. C., \& Herrera, F. (2018b). Multiple criteria decision making based on distance and similarity measures with double hierarchy hesitant fuzzy linguistic term sets. Computers \& Industrial Engineering, 126, 516-530. https://doi.org/10.1016/j.cie.2018.10.020

Herrera, F., Herrera-Viedma, E., \& Verdegay, J. L. (1996). A model of consensus in group decision making under linguistic assessments. Fuzzy Sets and Systems, 78(1), 73-87. https://doi.org/10.1016/0165-0114(95)00107-7

Herrera, F., Herrera-Viedma, E., \& Verdegay, J. L. (1995). A sequential selection process in group decision making with a linguistic assessment approach. Information Sciences, 85(4), 223-239. https://doi.org/10.1016/0020-0255(95)00025-K

Herrera, F., \& Martínez, L. (2000). A 2-tuple fuzzy linguistic representation model for computing with words. IEEE Transactions on Fuzzy Systems, 8, 746-752. https://doi.org/10.1109/91.890332

Herrera-Viedma, E., Alonso, S., Chiclana, F., \& Herrera, F. (2007). A consensus model for group decision making with incomplete fuzzy preference relations. IEEE Transactions on Fuzzy Systems, 15(5), 863-877.

https://doi.org/10.1109/TFUZZ.2006.889952

Karplus, K. P., \& Diederichs, K. (2012). Linking crystallographic model and data quality. Science, 336(6084), 1030-1033. https://doi.org/10.1126/science.1218231

Krishankumar, R., Subrajaa, L. S., Ravichandran, K. S., Kar, S., \& Saeid, A. B. (2019). A framework for multi-attribute group decision-making using double hierarchy hesitant fuzzy linguistic term set. International Journal of Fuzzy Systems. https://doi.org/10.1007/s40815-019-00618-w

Li, C. C., Dong, Y. C., Xu, Y. J., Chiclana, F., Herrera-Viedma, E., \& Herrera, F. (2019). An overview on managing additive consistency of reciprocal preference relations for consistency-driven decision making and fusion: taxonomy and future directions. Information Fusion, 52, 143-156. https://doi.org/10.1016/j.inffus.2018.12.004

Li, J., \& Wang, J. Q. (2018). Multi-criteria decision making with probabilistic hesitant fuzzy information based on expected multiplicative consistency. Neural Computing and Applications. https://doi.org/10.1007/s00521-018-3753-1

Li, J., Wang, J. Q., \& Hu, J. H. (2019). Consensus building for hesitant fuzzy preference relations with multiplicative consistency. Computers \& Industrial Engineering, 128, 387-400. https://doi.org/10.1016/j.cie.2018.12.051

Liao, H. C., Xu, Z. S., Zeng, X. J., \& Xu, D. L. (2015). An enhanced consensus reaching process in group decision making with intuitionistic fuzzy preference relations. Information Sciences, 329(C), 274-286. https://doi.org/10.1016/j.ins.2015.09.024
Liu, C., \& Xiong, W. (2018). China's real estate market (Working paper). National Bureau of Economic Research.

https://doi.org/10.3386/w25297

Luo, S. Z., Zhang, H. Y., Wang, J. Q., \& Li, L. (2019) Group decision-making approach for evaluating the sustainability of constructed wetlands with probabilistic linguistic preference relations. Journal of the Operational Research Society. https://doi.org/10.1080/01605682.2018.151080

Ma, L. C. (2016). A new group ranking approach for ordinal preferences based on group maximum consensus sequences. European Journal of Operational Research, 251(1), 171-181. https://doi.org/10.1016/j.ejor.2015.10.042

Mendel, J. M. (2007). Type-2 fuzzy sets and systems: an overview. IEEE Computational Intelligence Magazine, 2(2), 20-29. https://doi.org/10.1109/MCI.2007.380672

Millet, I. (1997). The effectiveness of alternative preference elicitation methods in the analytic hierarchy process. Journal of Multi-Criteria Decision Analysis, 6, 41-51.

https://doi.org/10.1002/(SICI)1099-1360(199701)6: $1<41::$ AID-MCDA122>3.0.CO;2-D

Montserrat-Adell, J., Xu, Z. S., Gou, X. J., \& Agell, N. (2019). Free double hierarchy hesitant fuzzy linguistic term sets: an application on ranking alternatives in GDM. Information Fusion, 47, 45-59. https://doi.org/10.1016/j.inffus.2018.07.002

Orlorski, S. A. (1978). Decision-making with a fuzzy preference relation. Fuzzy Sets and Systems, 3, 155-167. http://doi.org/10.1016/0165-0114(78)90001-5

Qin, J. D., Liu, X. W., \& Pedrycz, W. (2017). An extended TODIM multi-criteria group decision making method for green supplier selection in interval type-2 fuzzy environment. European Journal of Operational Research, 258(2), 626-638. https://doi.org/10.1016/j.ejor.2016.09.059

Rodríguez, R. M., Martínez, L., \& Herrera, F. (2012). Hesitant fuzzy linguistic terms sets for decision making. IEEE Transactions on Fuzzy Systems, 20, 109-119. https://doi.org/10.1109/TFUZZ.2011.2170076

Saaty, T. L. (1980). The analytical hierarchy process. New York: McGraw-Hill. https://doi.org/10.21236/ADA214804

Schumpeter, J. A. (1934). The theory of economic development: an inquiry into profits, capital, credit, interest, and the business cycle. Social Science Electronic Publishing, 25(1), 90-91.

Wang, H., Xu, Z. S., \& Zeng, X. J. (2018). Linguistic terms with weakened hedges: a model for qualitative decision making under uncertainty. Information Sciences, 433, 37-45. https://doi.org/10.1016/j.ins.2017.12.036

Wu, X. L., \& Liao, H. C. (2019). A consensus-based probabilistic linguistic gained and lost dominance score method. European Journal of Operational Research, 272(3), 1017-1027. https://doi.org/10.1016/j.ejor.2018.07.044

Wu, Z. B., \& Xu, J. P. (2016). Managing consistency and consensus in group decision making with hesitant fuzzy linguistic preference relations. Omega, 65(3), 28-40. https://doi.org/10.1016/j.omega.2015.12.005

Xia, M. M., \& Xu, Z. S. (2011). Some issues on multiplicative consistency of interval reciprocal relations. International Journal of Information Technology \& Decision Making, 10(6), 1043-1065. https://doi.org/10.1142/S0219622011004701

Xia, M. M., Xu, Z. S., \& Chen, J. (2013). Algorithms for improving consistency or consensus of reciprocal [0,1]-valued preference relations. Fuzzy Sets and Systems, 216, 108-133. https://doi.org/10.1016/j.fss.2012.09.016

Xia, M. M., Xu, Z. S., \& Wang, Z. (2014). Multiplicative consistency-based decision support system for incomplete linguistic 
preference relations. International Journal of Systems Science, 45(3), 625-636.

https://doi.org/10.1080/00207721.2012.724112

$\mathrm{Xu}, \mathrm{Z}$. S. (2005). Deviation measures of linguistic preference relations in group decision making. Omega, 33(3), 249-254. https://doi.org/10.1016/j.omega.2004.04.008

$\mathrm{Xu}, \mathrm{Z}$. S. (2007). Intuitionistic preference relations and their application in group decision making. Information Sciences, 177 , 2363-2379. https://doi.org/10.1016/j.ins.2006.12.019

$\mathrm{Xu}, \mathrm{Z}$. S. (2008). Group decision making based on multiple types of linguistic preference relations. Information Sciences, 178, 452-467. https://doi.org/10.1016/j.ins.2007.05.018

Xu, Z. S., Cai, X. Q., \& Szmidt, E. (2011). Algorithms for estimating missing elements of incomplete intuitionistic preference relations. International Journal of Intelligent Systems, 26(9), 787-813. https://doi.org/10.1002/int.20494

Yazdipour, R. (2011). Advances in entrepreneurial finance. New York: Springer. https://doi.org/10.1007/978-1-4419-7527-0

Yu, D. J., Xu, Z. S., \& Wang, W. (2018). Bibliometric analysis of fuzzy theory research in China: a 30-year perspective. Knowledge-Based Systems, 141, 188-199.

https://doi.org/10.1016/j.knosys.2017.11.018

Zacharakis, A. L., Mcmullen, J. S., \& Shepherd, D. A. (2007). Venture capitalists' decision making across three countries: an institutional theory perspective. Journal of International Business Studies, 38(5), 691-708.

https://doi.org/10.1057/palgrave. jibs.8400291

Zadeh, L. A. (1975). The concept of a linguistic variable and its application to approximate reasoning I. Information Sciences, 8, 199-249. https://doi.org/10.1016/0020-0255(75)90036-5
Zadeh, L. A. (2012). Computing with words: what is computing with words $(C W W)$ ? (pp. 1-40). Springer. https://doi.org/10.1007/978-3-642-27473-2

Zhang, W. K., Du, J., \& Tian, X. L. (2018). Finding a promising venture capital project with todim under probabilistic hesitant fuzzy circumstance. Technological and Economic Development of Economy, 24(5), 2026-2044.

https://doi.org/10.3846/tede.2018.5494

Zhang, Y. X., Xu, Z. S., Wang, H., \& Liao, H. C. (2016). Consistency-based risk assessment with probabilistic linguistic preference relation. Applied Soft Computing, 49, 817-833. https://doi.org/10.1016/j.asoc.2016.08.045

Zhang, X. Y., Zhang H. Y., \& Wang, J. Q. (2019). Discussing incomplete 2-tuple fuzzy linguistic preference relations in multi-granular linguistic MCGDM with unknown weight information. Soft Computing, 23(6), 2015-2032. https://doi.org/10.1007/s00500-017-2915-x

Zhou, W., \& Xu, Z. S. (2018). Probability calculation and element optimization of probabilistic hesitant fuzzy preference relations based on expected consistency. IEEE Transactions on Fuzzy Systems, 26(3), 1367-1378. https://doi.org/10.1109/TFUZZ.2017.2723349

Zhu, B., \& Xu, Z. S. (2014). Consistency measures for hesitant fuzzy linguistic preference relations. IEEE Transactions on Fuzzy Systems, 22(1), 35-45. https://doi.org/10.1109/TFUZZ.2013.2245136

Zhu, B., Xu, Z. S., \& Xu, J. P. (2014). Deriving a ranking from hesitant fuzzy preference relations under group decision making. IEEE Transactions on Cybernetics, 44(8), 1328-1337. https://doi.org/10.1109/TCYB.2013.2283021

\section{Appendix}

Proof of Theorem 1: I $\Leftrightarrow$ II. Based on Eq. (9), we obtain

$$
\begin{aligned}
& f\left(h_{S_{O_{i k}}^{N}}^{N(l)}\right) f\left(h_{S_{O_{k j}}^{N(l)}}^{N(l)}\right) f\left(h_{S_{O_{j i}}^{N(l)}}\right)=f\left(h_{S_{O_{k i}}^{N}}^{N(l)}\right) f\left(h_{S_{O_{j k}}^{N(l)}}\right) f\left(h_{S_{O_{i j}}^{N(l)}}^{N(l)}\right) \Leftrightarrow f\left(h_{S_{O_{i k}}}^{N(l)}\right) f\left(h_{S_{O_{k j}}}^{N(l)}\right)\left(1-f\left(h_{S_{O_{i j}}}^{N(l)}\right)\right)= \\
& \left(1-f\left(h_{S_{O_{i k}}^{N(l)}}\right)\right)\left(1-f\left(h_{S_{O_{k j}}^{N(l)}}^{N(j)}\right)\right) f\left(h_{S_{O_{i j}}^{N(l)}}^{N}\right) \\
& \Leftrightarrow f\left(h_{S_{O_{i j}}}^{N(l)}\right)=\frac{f\left(h_{S_{O_{i k}}}^{N(l)}\right) f\left(h_{S_{O_{k j}}}^{N(l)}\right)}{f\left(h_{S_{O_{i k}}^{N(l)}}^{N(l)}\right) f\left(h_{S_{O_{k j}}}^{N(l)}\right)+\left(1-f\left(h_{S_{O_{i k}}^{N}}^{N(l)}\right)\right)\left(1-f\left(h_{S_{O_{k j}}}^{N(l)}\right)\right)}
\end{aligned}
$$

I $\Leftrightarrow$ III. Based on Eq. (9), there is $f\left(h_{S_{O_{i k}}}^{N(l)}\right) f\left(h_{S_{O_{k j}}}^{N(l)}\right) f\left(h_{S_{O_{j i}}^{N(l)}}^{N(l)}\right)=f\left(h_{S_{O_{k i}}}^{N(l)}\right) f\left(h_{S_{O_{j k}}^{N(l)}}^{N(l)}\right) f\left(h_{S_{O_{i j}}}^{N(l)}\right), i, j, k=1,2, \ldots, m$. i.e.,

$$
\begin{aligned}
& f\left(h_{S_{O_{i 1}}}^{N(l)}\right) f\left(h_{S_{O_{1 j}}}^{N(l)}\right) f\left(h_{S_{O_{j i}}}^{N(l)}\right)=f\left(h_{S_{O_{1 i}}^{N(l)}}^{N(x}\right) f\left(h_{S_{O_{j 1}}}^{N(l)}\right) f\left(h_{S_{O_{i j}}}^{N(l)}\right), \quad i, j=1,2, \ldots, m \\
& f\left(h_{S_{O_{i 2}}}^{N(l)}\right) f\left(h_{S_{O_{2 j}}}^{N(l)}\right) f\left(h_{S_{O_{j i}}^{N(l)}}\right)=f\left(h_{S_{O_{2 i}}^{N(l)}}\right) f\left(h_{S_{O_{j 2}}}^{N(l)}\right) f\left(h_{S_{O_{i j}}}^{N(l)}\right), \quad i, j=1,2, \ldots, m
\end{aligned}
$$

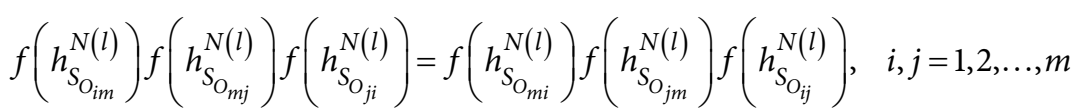


Multiplying Eqs (25)-(27), we obtain

$$
\begin{aligned}
& \left(f\left(h_{S_{O_{j i}}}^{N(l)}\right)\right)^{m} \prod_{k=1}^{m} f\left(h_{S_{O_{i k}}}^{N(l)}\right) f\left(h_{S_{O_{k j}}^{N}}^{N(l)}\right)=\left(f\left(h_{S_{O_{i j}}^{N(l)}}\right)\right)^{m} \prod_{k=1}^{m} f\left(h_{S_{O_{k i}}}^{N(l)}\right) f\left(h_{S_{O_{j k}}}^{N(l)}\right), \quad i, j=1,2, \ldots, m \\
& \Rightarrow\left(1-f\left(h_{S_{O_{i j}}}^{N(l)}\right)\right) \sqrt[m]{\prod_{k=1}^{m} f\left(h_{S_{O_{i k}}}^{N(l)}\right) f\left(h_{S_{O_{k j}}}^{N(l)}\right)}=f\left(h_{S_{O_{i j}}}^{N(l)}\right) \sqrt[m]{\prod_{k=1}^{m} f\left(h_{S_{O_{k i}}}^{N(l)}\right) f\left(h_{S_{O_{j k}}}^{N(l)}\right)}, \quad i, j=1,2, \ldots, m
\end{aligned}
$$

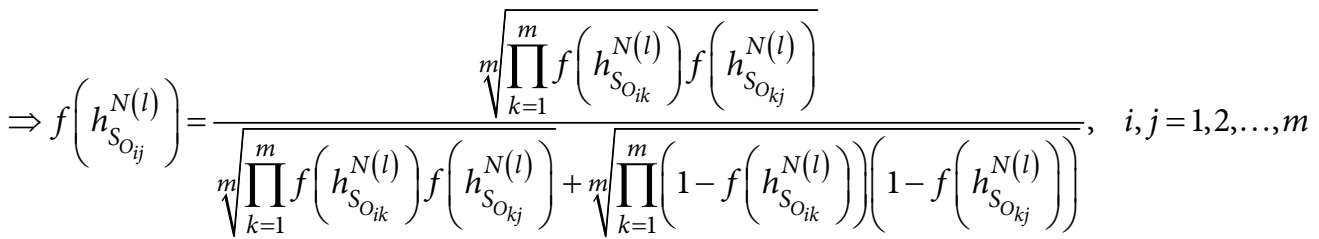

III $\Leftrightarrow$ I. Eq. (11) is equal to

$$
\left(f\left(h_{S_{O_{j i}}}^{N(l)}\right)\right)^{m} \prod_{z=1}^{m} f\left(h_{S_{O_{i z}}}^{N(l)}\right) f\left(h_{S_{O_{z j}}^{N(l)}}\right)=\left(f\left(h_{S_{O_{i j}}^{N(l)}}\right)\right)^{m} \prod_{z=1}^{m} f\left(h_{S_{O_{z i}}}^{N(l)}\right) f\left(h_{S_{O_{j z}}}^{N(l)}\right), \quad i, j=1,2, \ldots, m
$$

The following equations can be got:

$$
\begin{aligned}
& \left(f\left(h_{S_{O_{k i}}}^{N(l)}\right)\right)^{m} \prod_{z=1}^{m} f\left(h_{S_{O_{i z}}}^{N(l)}\right) f\left(h_{S_{O_{z k}}}^{N(l)}\right)=\left(f\left(h_{S_{O_{i k}}}^{N(l)}\right)\right)^{m} \prod_{z=1}^{m} f\left(h_{S_{O_{z i}}}^{N(l)}\right) f\left(h_{S_{O_{k z}}}^{N(l)}\right), \quad i, k=1,2, \ldots, m \\
& \left(f\left(h_{S_{O_{j k}}^{N(l)}}\right)\right)^{m} \prod_{z=1}^{m} f\left(h_{S_{O_{k z}}^{N(l)}}^{m}\right) f\left(h_{S_{O_{z j}}^{N(l)}}\right)=\left(f\left(h_{S_{O_{k j}}}^{N(l)}\right)\right)^{m} \prod_{z=1}^{m} f\left(h_{S_{O_{z k}}}^{N(l)}\right) f\left(h_{S_{O_{j z}}}^{N(l)}\right), \quad k, j=1,2, \ldots, m
\end{aligned}
$$

Multiplying Eqs (29) and (30):

$$
\left(f\left(h_{S_{O_{k i}}}^{N(l)}\right)\right)^{m}\left(f\left(h_{S_{O_{j k}}}^{N(l)}\right)\right)^{m} \prod_{z=1}^{m} f\left(h_{S_{O_{i z}}}^{N(l)}\right) f\left(h_{S_{O_{z j}}}^{N(l)}\right)=\left(f\left(h_{S_{O_{i k}}}^{N(l)}\right)\right)^{m}\left(f\left(h_{S_{O_{k j}}}^{N(l)}\right)\right)^{m} \prod_{z=1}^{m} f\left(h_{S_{O_{z i}}}^{N(l)}\right) f\left(h_{S_{O_{j z}}}^{N(l)}\right), \quad i, j=1,2, \ldots, m
$$

Dividing Eq. (28) by Eq. (31),

$$
\begin{aligned}
& \frac{\left(f\left(h_{S_{O_{k i}}}^{N(l)}\right)\right)^{m}\left(f\left(h_{S_{O_{j k}}}^{N(l)}\right)\right)^{m}}{\left(f\left(h_{S_{O_{j i}}^{N}}^{N(l)}\right)\right)^{m}}=\frac{\left(f\left(h_{S_{O_{i k}}}^{N(l)}\right)\right)^{m}\left(f\left(h_{S_{O_{k j}}}^{N(l)}\right)\right)^{m}}{\left(f\left(h_{S_{O_{i j}}^{N(l)}}^{m}\right)\right)^{m}}, i, j, k=1,2, \ldots, m \\
& \left(f\left(h_{S_{O_{k i}}^{N(l)}}\right)\right)^{m}\left(f\left(h_{S_{O_{j k}}^{N(l)}}\right)\right)^{m}\left(f\left(h_{S_{O_{i j}}^{N(l)}}\right)\right)^{m}=\left(f\left(h_{S_{O_{i k}}^{N(l)}}\right)\right)^{m}\left(f\left(h_{S_{O_{k j}}^{N}}^{N(l)}\right)\right)^{m}\left(f\left(h_{S_{O_{j i}}^{N}}^{N(l)}\right)\right)^{m}, i, j, k=1,2, \ldots, m
\end{aligned}
$$

which indicates that $\tilde{H}_{S_{O}}$ is multiplicative consistent. This completes the proof of Theorem 1.

Proof of Theorem 2: For $i, j, k=1,2, \ldots, m$, we have

$$
\begin{aligned}
& f\left(\hat{h}_{S_{O_{i k}}^{N(l)}}\right)=\frac{\sqrt[m]{\prod_{z=1}^{m} f\left(h_{S_{O_{i z}}}^{N(l)}\right) f\left(h_{S_{O_{z k}}}^{N(l)}\right)}}{\sqrt[m]{\prod_{z=1}^{m} f\left(h_{\left.S_{O_{i z}}^{N(l)}\right) f\left(h_{S_{O_{z k}}^{N(l)}}\right)}^{m}\right.}+\sqrt[m]{\prod_{z=1}^{m}\left(1-f\left(h_{S_{O_{i z}}}^{N(l)}\right)\right)\left(1-f\left(h_{S_{O_{z k}}}^{N(l)}\right)\right)}} \\
& f\left(\hat{h}_{S_{O_{k j}}^{N}}^{N(l)}\right)=\frac{\sqrt[m]{\prod_{z=1}^{m} f\left(h_{S_{O_{k z}}}^{N(l)}\right) f\left(h_{S_{O_{z j}}}^{N(l)}\right)}}{\sqrt[m]{\prod_{z=1}^{m} f\left(h_{S_{O_{k z}}^{N}}^{N(l)}\right) f\left(h_{S_{O_{z j}}^{N(l)}}^{N}\right)}+\sqrt[m]{\prod_{z=1}^{m}\left(1-f\left(h_{S_{O_{k z}}^{N(l)}}\right)\right)\left(1-f\left(h_{S_{O_{z j}}}^{N(l)}\right)\right)}}
\end{aligned}
$$


Let

$$
\begin{aligned}
& \mathbb{Z}_{i k}=\sqrt[m]{\prod_{z=1}^{m} f\left(h_{S_{O_{i z}}}^{N(l)}\right) f\left(h_{S_{O_{z k}}}^{N(l)}\right)}+\sqrt[m]{\prod_{z=1}^{m}\left(1-f\left(h_{S_{O_{i z}}}^{N(l)}\right)\right)\left(1-f\left(h_{S_{O_{z k}}}^{N(l)}\right)\right)} \\
& \mathbb{Z}_{k j}=\sqrt[m]{\prod_{z=1}^{m} f\left(h_{S_{O_{k z}}}^{N(l)}\right) f\left(h_{S_{O_{z j}}^{N(l)}}\right)}+\sqrt[m]{\prod_{z=1}^{m}\left(1-f\left(h_{S_{O_{k z}}^{N(l)}}\right)\right)\left(1-f\left(h_{S_{O_{z j}}^{N(l)}}^{N()}\right)\right)}
\end{aligned}
$$

Then,

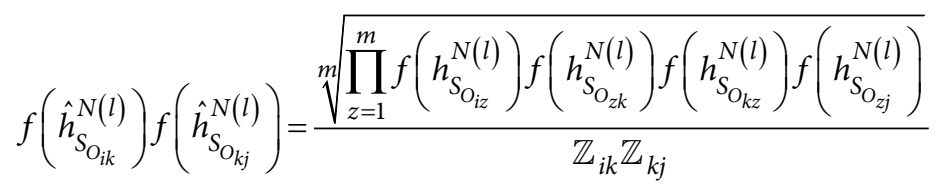

and

$$
\begin{aligned}
& f\left(\hat{h}_{S_{O_{i k}}}^{N(l)}\right) f\left(\hat{h}_{S_{O_{k j}}}^{N(l)}\right)+\left(1-f\left(\hat{h}_{S_{O_{i k}}}^{N(l)}\right)\right)\left(1-f\left(\hat{h}_{S_{O_{k j}}}^{N(l)}\right)\right)= \\
& \frac{\sqrt[m]{\prod_{z=1}^{m} f\left(h_{S_{O_{i z}}}^{N(l)}\right) f\left(h_{S_{O_{z k}}}^{N(l)}\right) f\left(h_{S_{O_{k z}}}^{N(l)}\right) f\left(h_{S_{O_{z j}}}^{N(l)}\right)}}{\mathbb{Z}_{i k} \mathbb{Z}_{k j}}\left(1-\frac{\sqrt[m]{\prod_{z=1}^{m} f\left(h_{S_{O_{i z}}}^{N(l)}\right) f\left(h_{S_{O_{z k}}}^{N(l)}\right)}}{\mathbb{Z}_{i k}}\right)\left(1-\frac{\sqrt[m]{\prod_{z=1}^{m} f\left(h_{S_{O_{k z}}}^{N(l)}\right) f\left(h_{S_{O_{z j}}}^{N(l)}\right)}}{\mathbb{Z}_{k j}}\right) \\
& \frac{\sqrt[m]{\prod_{z=1}^{m} f\left(h_{S_{O_{i z}}}^{N(l)}\right) f\left(h_{S_{O_{z k}}}^{N(l)}\right) f\left(h_{S_{O_{k z}}}^{N(l)}\right) f\left(h_{S_{O_{z j}}}^{N(l)}\right)}+\sqrt[m]{\prod_{z=1}^{m}\left(1-f\left(h_{S_{O_{i z}}}^{N(l)}\right)\right)\left(1-f\left(h_{S_{O_{z k}}}^{N(l)}\right)\right)\left(1-f\left(h_{S_{O_{k z}}}^{N(l)}\right)\right)\left(1-f\left(h_{S_{O_{z j}}}^{N(l)}\right)\right)}}{\mathbb{Z}_{i k} \mathbb{Z}_{k j}}= \\
& \prod_{z=1}^{m} f\left(h_{S_{O_{z k}}^{N(l)}}\right) f\left(h_{S_{O_{k z}}^{N(l)}}\right)=\prod_{z=1}^{m}\left(1-f\left(h_{\left.S_{O_{k z}}^{N(l)}\right)}\right)\right)\left(1-f\left(h_{S_{O_{z k}}^{N}}^{N(l)}\right)\right)=\prod_{z=1}^{m}\left(1-f\left(h_{S_{O_{z k}}^{N(l)}}\right)\right)\left(1-f\left(h_{S_{O_{k z}}^{N}}^{N(l)}\right)\right)
\end{aligned}
$$

Therefore,

$$
\begin{aligned}
& \frac{f\left(\hat{h}_{S_{O_{i k}}^{N(l)}}\right) f\left(\hat{h}_{S_{O_{k j}}^{N(l)}}\right)}{f\left(\hat{h}_{S_{O_{i k}}^{N(l)}}^{N}\right) f\left(\hat{h}_{S_{O_{k j}}^{N(l)}}^{N}\right)+\left(1-f\left(\hat{h}_{S_{O_{i k}}^{N(l)}}\right)\right)\left(1-f\left(\hat{h}_{S_{O_{k j}}^{N(l)}}\right)\right)}= \\
& \sqrt[m]{\prod_{z=1}^{m} f\left(h_{S_{O_{i z}}}^{N(l)}\right) f\left(h_{S_{O_{z k}}^{N(l)}}\right) f\left(h_{S_{O_{k z}}^{N(l)}}\right) f\left(h_{S_{O_{z j}}^{N(l)}}\right)} \\
& \sqrt[m]{\prod_{z=1}^{m} f\left(h_{S_{O_{i z}}}^{N(l)}\right) f\left(h_{S_{O_{z k}}}^{N(l)}\right) f\left(h_{S_{O_{k z}}}^{N(l)}\right) f\left(h_{S_{O_{z j}}}^{N(l)}\right)}+\sqrt[m]{\prod_{z=1}^{m}\left(1-f\left(h_{S_{O_{i z}}^{N(l)}}\right)\right)\left(1-f\left(h_{S_{O_{z k}}}^{N(l)}\right)\right)\left(1-f\left(h_{S_{O_{k z}}^{N(l)}}\right)\right)\left(1-f\left(h_{S_{O_{z j}}^{N(l)}}\right)\right)}= \\
& \frac{\sqrt[m]{\prod_{z=1}^{m} f\left(h_{S_{O_{i z}}}^{N(l)}\right) f\left(h_{S_{O_{z j}}^{N(l)}}\right)}}{\sqrt[m]{\prod_{z=1}^{m} f\left(h_{S_{O_{i z}}^{N(l)}}^{N(l)} f\left(h_{S_{O_{z j}}}^{N(l)}\right)\right.}+\sqrt[m]{\prod_{z=1}^{m}\left(1-f\left(h_{\left.S_{O_{i z}}^{N(l)}\right)}^{N}\right)\left(1-f\left(h_{S_{O_{z j}}}^{N(l)}\right)\right)\right.}}=f\left(\hat{h}_{S_{O_{i j}}^{N(l)}}\right)
\end{aligned}
$$

Additionally, $f\left(\hat{h}_{S_{O_{i j}}^{N}}^{N(l)}\right)+f\left(\hat{h}_{S_{O_{j i}}^{N}}^{N(l)}\right)$ 


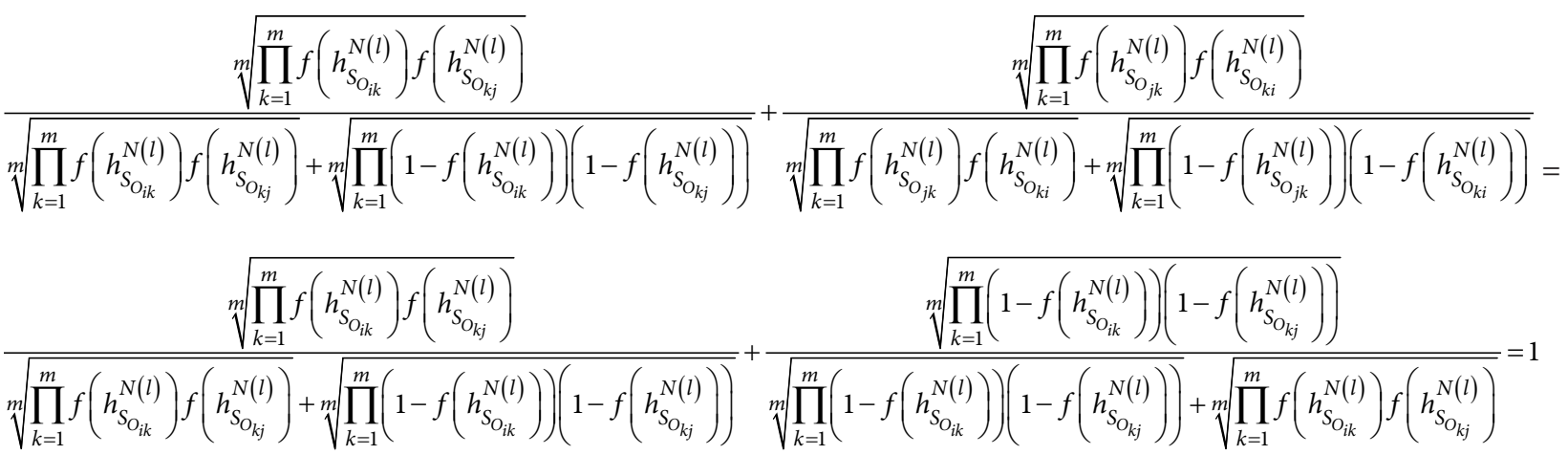

The proof of Theorem 2 is completed.

Proof of Theorem 3: Since the repaired DHHFLPR $\left(\tilde{H}_{S_{O}}\right)^{(\mathbb{Z})}=\left(\left(h_{S_{O_{i j}}}\right)_{m \times m}\right)^{(\mathbb{Z})}=\left(\left(\left\{h_{S_{O_{i j}}}^{(l)} \mid l=1,2, \ldots, \# h_{S_{O_{i j}}}\right\}\right)_{m \times m}\right)^{(\mathbb{Z})}$ is constructed by the DMs' preferences with an IV-DHHFLPR, $\tilde{H}_{\bar{S}_{\bar{O}}}=\left(\left(h_{\bar{S}_{\bar{S}_{i j}}}\right)_{m \times m}\right)^{(\mathbb{Z})}$. Then we have

$\left(h_{S_{O_{i j}}}^{(l)}\right)^{(\mathbb{Z}+1)} \in\left[\min \left\{\left(h_{S_{O_{i j}}}^{(l)}\right)^{(\mathbb{Z})}, \hat{h}_{S_{O_{i j}}^{N(l)}}\right\}, \max \left\{\left(h_{S_{O_{i j}}}^{(l)}\right)^{(\mathbb{Z})}, \hat{h}_{S_{O_{i j}}^{N(l)}}\right\}\right]$

Thus,

$\mid f\left(\left(h_{S_{O_{i j}}^{(l)}}^{(\mathbb{Z}+1)}\right)-f\left(\hat{h}_{S_{O_{i j}}^{N(l)}}\right)|\leq| f\left(\left(h_{S_{O_{i j}}}^{(l)}\right)^{(\mathbb{Z})}\right)-f\left(\hat{h}_{S_{O_{i j}}^{N(l)}}\right) \mid\right.$

Based on Eqs (34) and (35), and Eq. (15), we have

$1-\left(\frac{2}{m(m-1)} \sum_{i<j}^{m}\left(\frac{1}{L} \sum_{l=1}^{L}\left(f\left(\left(h_{S_{O_{i j}}}^{(l)}\right)^{(\mathbb{Z}+1)}\right)-f\left(\hat{h}_{S_{O_{i j}}^{N}(l)}\right)\right)^{2}\right)\right)^{1 / 2} \geq 1-\left(\frac{2}{m(m-1)} \sum_{i<j}^{m}\left(\frac{1}{L} \sum_{l=1}^{L}\left(f\left(\left(h_{S_{O_{i j}}}^{(l)}\right)^{(\mathbb{Z})}\right)-f\left(\hat{h}_{S_{O_{i j}}^{N(l)}}\right)\right)^{2}\right)\right)^{1 / 2}$

Then, we get $C I\left(\left(\tilde{H}_{S_{O}}\right)^{(\mathbb{Z}+1)}\right) \geq C I\left(\left(\tilde{H}_{S_{O}}\right)^{(\mathbb{Z})}\right)$, i.e., $C I\left(* \tilde{H}_{S_{O}}\right) \geq C I\left(\tilde{H}_{S_{O}}\right)$. This completes the proof of Theorem 3. Proof of Theorem 4: Since $h_{S_{O_{q}}}^{+}=\max _{i} h_{S_{O_{q}}}^{i}$ and $h_{S_{O_{q}}}^{-}=\min _{i} h_{S_{O_{q}}}^{i}$, then we have $\left|E\left(h_{S_{O_{q}}}^{+}\right)-E\left(h_{S_{O_{q}}}^{-}\right)\right| \geq\left|E\left(h_{S_{O_{q}}}^{+}\right)-E\left(h_{S_{O_{q}}}^{i}\right)\right|$. Thus, we can obtain $d\left(h_{S_{O_{q}}^{+}}^{+}, h_{S_{O_{q}}}^{-}\right) \geq d\left(h_{S_{O_{q}}^{+}}^{+}, h_{S_{O_{q}}}^{i}\right), i=1,2, \ldots, m$. Therefore, $d\left(h_{S_{O_{q}}}^{+}, h_{S_{O_{q}}}^{i}\right) / d\left(h_{S_{O_{q}}}^{+}, h_{S_{O_{q}}}^{-}\right) \leq 1$. Let $\Delta_{q}^{i}=d\left(h_{S_{O_{q}}}^{+}, h_{S_{O_{q}}}^{i}\right) / d\left(h_{S_{O_{q}}}^{+}, h_{S_{O_{q}}}^{-}\right)-\frac{1}{m} \sum_{i=1}^{m}\left(d\left(h_{S_{O_{q}}^{+}}^{+}, h_{S_{O_{q}}}^{i}\right) / d\left(h_{S_{O_{q}}}^{+}, h_{S_{O_{q}}}^{-}\right)\right), \quad q=1,2$

Then $\Delta_{q}^{i} \in[-1,1]$. Eq. (18) can be transformed to $C\left(H_{S_{O_{1}}}, H_{S_{O_{2}}}\right)=\sum_{i=1}^{m}\left(\Delta_{1}^{i} \times \Delta_{2}^{i}\right) /\left(\sqrt{\sum_{i=1}^{m}\left(\Delta_{1}^{i}\right)^{2}} \times \sqrt{\sum_{i=1}^{m}\left(\Delta_{2}^{i}\right)^{2}}\right)$.

(1) When $i=1, C\left(H_{S_{O_{1}}}, H_{S_{O_{2}}}\right)=1 \in[-1,1]$. 
(2) When $i=2, C\left(H_{S_{O_{1}}}, H_{S_{O_{2}}}\right)=\Delta_{1}^{1} \Delta_{2}^{1}+\Delta_{1}^{2} \Delta_{2}^{2} /\left(\sqrt{\left(\Delta_{1}^{1}\right)^{2}+\left(\Delta_{1}^{2}\right)^{2}} \times \sqrt{\left(\Delta_{2}^{1}\right)^{2}+\left(\Delta_{2}^{2}\right)^{2}}\right)$. Then,

$C^{2}\left(H_{S_{O_{1}}}, H_{S_{O_{2}}}\right)=\left(\left(\Delta_{1}^{1} \Delta_{2}^{1}\right)^{2}+\left(\Delta_{1}^{2} \Delta_{2}^{2}\right)^{2}+2 \Delta_{1}^{1} \Delta_{2}^{1} \Delta_{1}^{2} \Delta_{2}^{2}\right) /\left(\left(\Delta_{1}^{1} \Delta_{2}^{1}\right)^{2}+\left(\Delta_{1}^{1} \Delta_{2}^{2}\right)^{2}+\left(\Delta_{1}^{2} \Delta_{2}^{1}\right)^{2}+\left(\Delta_{1}^{2} \Delta_{2}^{2}\right)^{2}\right)$

According to average value inequality, we get

$\left(\Delta_{1}^{1} \Delta_{2}^{1}\right)^{2}+\left(\Delta_{1}^{2} \Delta_{2}^{2}\right)^{2}+2 \Delta_{1}^{1} \Delta_{2}^{1} \Delta_{1}^{2} \Delta_{2}^{2} \leq\left(\Delta_{1}^{1} \Delta_{2}^{1}\right)^{2}+\left(\Delta_{1}^{1} \Delta_{2}^{2}\right)^{2}+\left(\Delta_{1}^{2} \Delta_{2}^{1}\right)^{2}+\left(\Delta_{1}^{2} \Delta_{2}^{2}\right)^{2}$

Then $C^{2}\left(H_{S_{\mathrm{O}_{1}}}, H_{S_{\mathrm{O}_{2}}}\right) \leq 1$, namely, $C\left(H_{S_{\mathrm{O}_{1}}}, H_{S_{\mathrm{O}_{2}}}\right) \in[-1,1]$.

(3) Suppose that $C\left(H_{S_{O_{1}}}, H_{S_{O_{2}}}\right) \in[-1,1]$ when $i=m$.

(4) When $i=m+1$, we have

$$
C\left(H_{S_{\mathrm{O}_{1}}}, H_{S_{\mathrm{O}_{2}}}\right)=\sum_{i=1}^{m}\left(\Delta_{1}^{i} \Delta_{2}^{i}\right)+\left(\Delta_{1}^{m+1} \Delta_{2}^{m+1}\right) /\left(\sqrt{\sum_{i=1}^{m}\left(\Delta_{1}^{i}\right)^{2}+\left(\Delta_{1}^{m+1}\right)^{2}} \times \sqrt{\sum_{i=1}^{m}\left(\Delta_{2}^{i}\right)^{2}+\left(\Delta_{2}^{m+1}\right)^{2}}\right)
$$

Let $a=\sum_{i=1}^{m}\left(\Delta_{1}^{i} \Delta_{2}^{i}\right), b=\sum_{i=1}^{m}\left(\Delta_{1}^{i}\right)^{2}$, and $c=\sum_{i=1}^{m}\left(\Delta_{2}^{i}\right)^{2}$, where $a \in[-1,1] ; b, c \in[0,1]$. Clearly, we have $b+c \geq 2 a$ and $b c \geq a^{2}$.

Then, $C^{2}\left(H_{S_{O_{1}}}, H_{S_{O_{2}}}\right)=a^{2}+2 a \Delta_{1}^{m+1} \Delta_{2}^{m+1}+\left(\Delta_{1}^{m+1} \Delta_{2}^{m+1}\right)^{2} /\left(b c+b\left(\Delta_{2}^{m+1}\right)^{2}+c\left(\Delta_{1}^{m+1}\right)^{2}+\left(\Delta_{1}^{m+1} \Delta_{2}^{m+1}\right)^{2}\right)$, and $a^{2}+2 a \Delta_{1}^{m+1} \Delta_{2}^{m+1}+\left(\Delta_{1}^{m+1} \Delta_{2}^{m+1}\right)^{2} \leq\left(b c+b\left(\Delta_{2}^{m+1}\right)^{2}+c\left(\Delta_{1}^{m+1}\right)^{2}+\left(\Delta_{1}^{m+1} \Delta_{2}^{m+1}\right)^{2}\right)$

Therefore, $C^{2}\left(H_{S_{\mathrm{O}_{1}}}, H_{S_{\mathrm{O}_{2}}}\right) \leq 1$, namely, $C\left(H_{S_{\mathrm{O}_{1}}}, H_{S_{\mathrm{O}_{2}}}\right) \in[-1,1]$.

According to the above proof, we get $C\left(H_{S_{\mathrm{O}_{1}}}, H_{S_{\mathrm{O}_{2}}}\right) \in[-1,1]$ for all $i$. This completes the proof. - 